\title{
Impact of participating in global value chain on the carbon dioxide emissions of China's equipment manufacturing industry
}

Yan Li

Dalian Maritime University

Xinxin Xia ( $2285008896 @ q q . c o m$ )

Dalian Maritime University https://orcid.org/0000-0002-1621-6215

Qingbo Huang

Dalian Maritime University

\section{Research Article}

Keywords: Global value chain, GVC production length, CO2 emission, Equipment manufacturing industry, CO2 emission effect model, STIRPAT model

Posted Date: April 2nd, 2021

DOI: https://doi.org/10.21203/rs.3.rs-315820/v1

License: (c) (i) This work is licensed under a Creative Commons Attribution 4.0 International License. Read Full License 
1 Impact of participating in global value chain on the carbon dioxide emissions of

2 China's equipment manufacturing industry

3

\author{
Yan Li $・$ Xinxin Xia $・$ Qingbo Huang ${ }^{1}$
}

\title{
Abstract
}

As the pillar industry in China's post-industrial era, the equipment manufacturing industry has played an important role of providing technical equipment for downstream industries, which also brought about a substantial increase in $\mathrm{CO}_{2}$ emissions. Therefore, in order to find ways to reduce the carbon dioxide emissions of the equipment manufacturing industry, this paper based on the global value chain production length decomposition model, improved the $\mathrm{CO}_{2}$ emission effect model and the STIRPAT model to study the different impact of the GVC production length on the $\mathrm{CO}_{2}$ emissions of China's equipment manufacturing industry under different GVC participation modes. The study found that extending GVC production length can effectively reduce $\mathrm{CO}_{2}$ emissions, and the $\mathrm{CO}_{2}$ reduction effect of the simple GVC production length is the most significant. Besides, with the extension of the GVC production length, the $\mathrm{CO}_{2}$ emissions of high-tech industries have decreased, while the $\mathrm{CO}_{2}$ emissions of medium-technology industries have increased. In addition, the improvements of policy regulations, factor structure and foreign investment will also reduce $\mathrm{CO}_{2}$ emissions, but the expansion of production scale and R\&D investment will increase $\mathrm{CO}_{2}$ emissions.

Keywords Global value chain $\cdot \mathrm{GVC}$ production length $\bullet \mathrm{CO}_{2}$ emission $\bullet$ Equipment manufacturing industry $\bullet \mathrm{CO}_{2}$ emission effect model $\bullet \mathrm{STIRPAT}$ model

\section{Introduction}

In the process of China's industrialization, China's equipment manufacturing industry assumes an important function of providing equipment and technical means for downstream industries (Liu and Zhu 2019), participating in the Global Value Chain (GVC) has greatly improved the level of production technology and production efficiency, which has brought huge economic profits and technical experience returns. But at the same time, because China's equipment manufacturing industry has a low degree of participation in GVC and is a resource-consuming industry (Wang et al. 2021), it has long become a major $\mathrm{CO}_{2}$ emitter in China (Guy et al. 2020). Since 2000, its $\mathrm{CO}_{2}$ emissions have accounted for about $9 \%$ of China's total $\mathrm{CO}_{2}$ emissions, and is about 10 times the level of $\mathrm{CO}_{2}$ emissions in industrialized countries (such as the United Kingdom, Germany, etc.), which is much higher than the average level of $\mathrm{CO}_{2}$ emissions of the global equipment manufacturing industry. Therefore, in the context of China's accelerated implementation of the " $1+X$ " planning system of "Made in China 2025", the equipment manufacturing industry must take the "green" path of independent innovation and sustainable development to achieve the transformation of high-quality and low- $\mathrm{CO}_{2}$ production. Hereto,

\footnotetext{
${ }^{1}$ School of Maritime Economics and Management, Dalian Maritime University, Dalian 116026, Liaoning, China;

Qingbo Huang: Email: huangqingbo@dlmu.edu.cn, Tel.: 86 +13079812778; Yan Li: Email: lilyyan@dlmu.edu.cn; Xinxin Xia: Email: 2285008896@qq.com.
} 
what impact will the deepening of participation in GVC have on the $\mathrm{CO}_{2}$ emissions of China's equipment manufacturing industry? Will the different modes of participating in GVC have different impact? What other factors also affect the $\mathrm{CO}_{2}$ emission of China's equipment manufacturing industry? The solution of the above problems will provide strong theoretical support for China's equipment manufacturing industry to find a path of low- $\mathrm{CO}_{2}$ development from the perspective of GVC.

To answer the above questions, the remainder of this paper is structured as follows: The "Literature review" section briefly reviews the current literature. The "Theoretical model" section presents the reasoning of the $\mathrm{CO}_{2}$ emission effect model with the participation of the GVC. The "Methodology and data" section depicts the GVC production length decomposition model and the econometric model of STIRPAT model, and affords data sources. The "Empirical results" section analyzes the regression outcomes of panel data of the equipment manufacturing industry from 2000 to 2014. Finally, the "Conclusions and policy implications" section provides the conclusions and targeted policy suggestions.

\section{Literature review}

At present, domestic and foreign scholars have few researches on the impact of participating in GVC on the $\mathrm{CO}_{2}$ emissions of equipment manufacturing industry. Relevant research mostly stays at the level of manufacturing industry. Besides, the measurement methods of GVC and research conclusions are also quite different. Through combing the related literature, this paper found that the measurement indicators of GVC are mainly divided into three aspects: GVC position index, GVC participation index and GVC production length. The research conclusions mainly include the following three points.

First of all, participating in GVC will increase the $\mathrm{CO}_{2}$ emissions of equipment manufacturing industry. The reason is that the low GVC participating degree of China's manufacturing industry is harmful for energy-saving and $\mathrm{CO}_{2}$-reduction. The expansion of low-end production activities has promoted the increase of $\mathrm{CO}_{2}$ emissions. Based on GVC position index, although China's manufacturing industry has improved trade competitiveness and basically shows an upward trend in GVC (Wei and Zhang 2020), it is difficult for China's equipment manufacturing industry to escape the development dilemma brought by the "low-end lock-in" of GVC (Chen and Wang 2015), which have aggravated the pollution problem caused by $\mathrm{CO}_{2}$ emissions (Sun and Du 2020), the phenomenon is particularly obvious in capital and technology-intensive industries (Wang 2014). Moreover, in terms of the GVC participation index, China's equipment manufacturing industry has a very high degree of "backward participation" in the GVC (Pan 2019), which requires more energy and resource input (Zhao et al. 2020), and makes $\mathrm{CO}_{2}$ emissions increase (Chang et al. 2020). Meanwhile on the basis of the GVC production length, after the extension of the GVC production length and the expansion of the scale of processing trade, the $\mathrm{CO}_{2}$ emissions generated by the manufacturing industry will also increase before the widespread application of cleaner production technologies (Zhao and Yang 2020). The low participating degree of China's manufacturing industry reflects the characteristics of weak technology ( $\mathrm{Li}$ and Yuan 2016), poor factor structure ( $\mathrm{Lu}$ et al. 2018), and strong mass production demand (Kang 2018; Edger 2020), which are also important factors to promote the increase of $\mathrm{CO}_{2}$ emissions (Xie et al. 2018).

Secondly, participating in GVC will decrease the $\mathrm{CO}_{2}$ emissions of equipment manufacturing 
industry. With the improvement of the GVC participation level of China's manufacturing, its technological level will continue to improve (Zhang and Gallagher 2016), and the output structure will continue to be optimized (Zhang et al. 2020). These value creation factors will enhance the impact on environmental sustainability (Stock et al. 2018), and provide a powerful boost to the energy saving and $\mathrm{CO}_{2}$ emission reduction of China's manufacturing industry. The climb of the GVC position is conducive to reducing manufacturing $\mathrm{CO}_{2}$ emissions (Zhang et al. 2018). Then, in the process of participating in GVC, China will improve the clean technology level of enterprises through imitation, learning and secondary innovation, urge enterprises to move upstream of GVC, and reduce environmental pollution (Cai et al. 2020). The rise of GVC participation index based on simple mode and complex mode can reduce China's $\mathrm{CO}_{2}$ emissions of production (Hao et al. 2020), and the rise of GVC participation index in high-tech manufacturing has a more significant $\mathrm{CO}_{2}$ emission reduction effect (Chang et al. 2020). Furthermore, the industrial structure upgrading effect brought about by the extension of the GVC production length is helpful for reducing the $\mathrm{CO}_{2}$ emissions of the manufacturing industry (Zhao and Yang 2020). As Chinese manufacturing industry is deeply participating in GVC, the continuous improvement of technology ( $\mathrm{Wu}$ and Pan 2018), the gradual optimization of the factor structure (Yu and Tian 2019), and the increasingly stringent environmental regulations (Zhang and Wei 2014) will significantly reduce $\mathrm{CO}_{2}$ emissions in China's production (Lan and Xia 2020; Chuanwang et al. 2019) .

The third conclusion is the U-shaped relationship between $\mathrm{GVC}$ and $\mathrm{CO}_{2}$ emissions. According to the Environmental Kuznets Curve (EKC) model (Grossman and Krueger 1995), domestic and foreign scholars have studied the $\mathrm{CO}_{2}$ emission reduction effects of manufacturing industry, and found that as the participating degree of GVC deepens, $\mathrm{CO}_{2}$ emissions will also show a U-shaped change. For example, when the economy is at the low end of the GVC position index, due to the scale effect and the industrial structure effect, the rise of the GVC position index has a promoting effect on $\mathrm{CO}_{2}$ emissions. But with the development of technology, production gradually shifts to a high value-added and low $\mathrm{CO}_{2}$-emission mode, The level of $\mathrm{CO}_{2}$ emissions will show a downward trend (Xu et al. 2020). Meanwhile, the $\mathrm{CO}_{2}$ emission reduction effect of GVC participation index will continue to be weaken. This is because when the leader country of the GVC see the development of the middle and downstream countries as a threat, they will prevent them from achieving GVC upgrades through technical barriers and other methods, and then lock the middle and downstream countries in the low-end GVC production links with high $\mathrm{CO}_{2}$ emissions (Humphrey and Schmitz 2010), which will turn the GVC into "global pollution chains" (Duan et al. 2020), in the end, the $\mathrm{CO}_{2}$ emission reduction effect of participating in GVC will be suppressed (Cai et al. 2020). At the same time, there is also an inverted U-shaped relationship between the GVC production length and $\mathrm{CO}_{2}$ emissions. Although China has passed the turning point, Chinese manufacturing has already paid huge environmental costs in the process of participating in GVC ( $\mathrm{Su}$ and Thomson 2016), which means that to a certain extent, participating in GVC is a stumbling block on the road to $\mathrm{CO}_{2}$ emission reduction (Lafang et al. 2020). In addition, affected by the participating in GVC, the technological level (Xie 2018), production scale (Grossman and Krueger 1995), factor structure (Huizheng et al. 2020), and output structure (Yuan et al. 2017) of China's equipment manufacturing industry may also show a U-shaped relationship with $\mathrm{CO}_{2}$ emissions .

In summary, the research on the impact of participating in $\mathrm{GVC}$ on manufacturing $\mathrm{CO}_{2}$ emissions has yielded fruitful results. However, the existing research still has three points that need to be 
expanded. First, the measurement methods for the degree of participating of GVC mainly stay in the two aspects of GVC position index and GVC participation index, it is impossible to comprehensively and scientifically describe the participating degree of GVC (Yuan and Qi et al. 2019). Secondly, it is still not clear enough of the $\mathrm{CO}_{2}$ emission effect model of GVC, and the internal links between $\mathrm{CO}_{2}$ emissions and related indicators need to be clarified. Lastly, few studies have explored the specific impact of participating in $\mathrm{GVC}$ on $\mathrm{CO}_{2}$ emissions from the perspective of sub-sector of equipment manufacturing industry.

Based on this, our study contributes to the previous literature in the following three aspects: (1) According to the global input-output table, the GVC production length is decomposed from the perspectives of the destination and the source of the value-added. And we distinguish the different concepts of the GVC production length, GVC simple production length, the GVC production length returned to the exporting country and the pure foreign GVC production length, which will help to comprehensively describe the situation of the equipment manufacturing industry in GVC and analyze the different results caused by different participating modes. (2) We update the analysis of environmental pollution and supply models, establish a $\mathrm{CO}_{2}$ emission effect model of the GVC, obtain relevant economic indicators affecting $\mathrm{CO}_{2}$ emissions. (3) We apply the $\mathrm{CO}_{2}$ emission effect model of GVC to a specific industry level, and deeply study the relationship between the GVC participation degree and the $\mathrm{CO}_{2}$ emissions of the equipment manufacturing industry, which will help to find the effective measures to achieve $\mathrm{CO}_{2}$ emission reduction targets while deeply participating in the GVC.

\section{Theoretical model}

Refer to the environmental pollution and supply model constructed by Antweiler et al. (2001), this part bases on the theory of perfect competition, introduces the effect function of the impact of GVC on production, and constructs a $\mathrm{CO}_{2}$ emission effect model with participating in GVC.

\section{Now suppose:}

(1) There are only two industries in the world, namely industry 1 and industry 2, of which industry 1 is a high- $\mathrm{CO}_{2}$ industry and industry 2 is other industries. Then the world only produces two products, that is, industry 1 produces product $X$ and industry 2 produces product $Y$. In addition, the production process of the two types of products obeys the principle of constant return to scale.

(2) Product $X$ is a high- $\mathrm{CO}_{2}$ product, that is, the production of product $X$ will discharge a large amount of pollutants. $Y$ is a low- $\mathrm{CO}_{2}$ product, that is, the production of $Y$ product does not emit any pollutants.

(3) Pollutants only consider $\mathrm{CO}_{2}$ emissions and ignore other environmental effects.

(4) The production only need two factors, they are labor $(L)$ and capital $(K)$.

(5) In an open economy with complete market competition, both industry 1 and industry 2 participate in the international division of labor.

Suppose the production function of potential output in the economy is:

$$
S=F(K, L)
$$


In Equation (1), $F$ is the production function, $S$ is the total output of the industry, $K$ is the capital input, and $L$ is the labor input. Since the production of products will be affected by internal and external elements, the actual output will be lower than the potential output. Therefore, assume that the internal element that affects production is only the factor input ratio $(G)$, that is, the ratio of capital input $(K)$ to labor input $(L)$; the external element that affects production is only the government's regulation of reducing $\mathrm{CO}_{2}$ emissions, in this case, $\mathrm{r}$ represents the rate of decrease of output. Since the input of reducing $\mathrm{CO}_{2}$ emissions will inhibit the increase of $\mathrm{CO}_{2}$ emissions, the level of $\mathrm{CO}_{2}$ emissions $\left(\mathrm{CO}_{2}\right.$ emissions per unit of output) can be expressed as $\varphi(r)=\frac{1}{T}(1-r)^{\frac{1}{\alpha}}$. Among them, $\varphi(r)$ is a decreasing function of $r$; the reciprocal form of production technology level $(T)$ represents the inhibitory effect of technological improvements on $\mathrm{CO}_{2}$ emissions; and $0<\alpha<1, \varphi^{\prime}(r)<0, \varphi^{\prime \prime}(r)>0$.

Then the actual production function of product $X$ is:

$$
S_{X}=G_{X}(1-r) F\left(K_{X}, L_{X}\right)
$$

The $\mathrm{CO}_{2}$ emissions during the production of product is:

$$
C=\varphi(r) F\left(K_{X}, L_{X}\right)=\frac{1}{T}(1-r)^{\frac{1}{\alpha}} F\left(K_{X}, L_{X}\right)
$$

Since industry 1 participates in the international division of production when producing $X$, the effect of participating in GVC on production is $\omega(V)$. At this time, the actual production function of product $X$ is:

$$
S_{X}=G_{X}(1-r) F\left(K_{X}, L_{X}\right) \omega(V)
$$

Incorporating formula 3 into formula 4 , the relationship between actual production level of product $X$ and $\mathrm{CO}_{2}$ emissions can be obtained:

$$
S_{X}=G_{X}(T C)^{\alpha} F\left(K_{X}, L_{X}\right)^{1-\alpha} \omega(V)
$$

Since $\mathrm{CO}_{2}$ emissions will cause negative externalization to the society, corresponding opportunity costs must be paid, so the tax rate for $\mathrm{CO}_{2}$ emissions is set to $\gamma$. According to the principle of minimizing the cost of enterprises, under normal circumstances, enterprises will choose the optimal arrangement of potential output and $\mathrm{CO}_{2}$ emission levels to achieve the lowest production cost of product. So we can construct the following function:

$$
\begin{aligned}
& \min _{C}\left\{E\left(E_{K}, E_{L}\right) F\left(K_{X}, L_{X}\right)+\gamma T C\right\} \\
& \text { s.t. } G_{X}(T C)^{\alpha} F\left(K_{X}, L_{X}\right)^{1-\alpha} \omega(V)=1
\end{aligned}
$$

Among them, $E\left(E_{K}, E_{L}\right)$ is the unit production cost of the potential output of product $X$, and $E_{K}, E_{L}$ is the production cost of capital and labor respectively.

By constructing a Lagrangian function, we can obtain the derivation of $\mathrm{CO}_{2}$ emission $C$ and output $F\left(K_{X}, L_{X}\right)$ respectively:

$$
\begin{aligned}
& \gamma T=-\alpha \theta G_{X} T^{\alpha} C^{\alpha-1} F\left(K_{X}, L_{X}\right)^{1-\alpha} \omega(V) \\
& E=-(1-\alpha) \theta G_{X} T^{\alpha} C^{\alpha} F\left(K_{X}, L_{X}\right)^{-\alpha} \omega(V)
\end{aligned}
$$

Among them, $\theta$ is the Lagrangian multiplier. Then divide the two formulas in Equation (7) to obtain the cost minimization conditions for the production of $X$ products by the enterprise.

$$
E=\frac{\gamma(1-\alpha) T C}{\alpha F\left(K_{X}, L_{X}\right)}
$$


Under perfectly competitive market conditions, the result of market competition is in line with Pareto optima. Then the net profit of the production of $X$ product must be zero, so the profit function of $X$ product is set as $\Pi=P_{X} S_{X}-E F\left(K_{X}, L_{X}\right)-\gamma T C$, where $P_{X}$ is the relative price of $X$ product relative to $Y$ product, and the price of $Y$ product is defined as 1 , we can obtain:

$$
P_{X} S_{X}=E F\left(K_{X}, L_{X}\right)+\gamma T C
$$

Combining Equation (8) with Equation (9), we obtain:

$$
S_{X}=\frac{\gamma T C}{\alpha P_{X}}
$$

Then, the $\mathrm{CO}_{2}$ emission level is:

$$
\varphi(r)=\frac{C}{S_{X}}=\frac{\alpha P_{X}}{\gamma T}
$$

The $\mathrm{CO}_{2}$ emission function in Equation (3) can be rewritten as:

$$
C=\varphi(r) F\left(K_{X}, L_{X}\right)=\frac{\alpha P_{X}}{\gamma T} \cdot \frac{S_{X}}{G_{X}(1-r) \omega(V)}
$$

Equation (12) is the decomposition model of the $\mathrm{CO}_{2}$ emission effect of product $X$ participating in the GVC. After taking the logarithm of both sides, we obtain:

$$
\ln C=\ln \left(\frac{\alpha P_{X}}{\gamma}\right)-\ln T+\ln S_{X}-\ln G_{X}-\ln (1-r)-\ln \omega(V)
$$

Among them, $\ln \left(\frac{\alpha P_{X}}{\gamma}\right)$ is $(S)$ is positive, which means that as the production scale expands, $\mathrm{CO}_{2}$ emissions will increase; the sign of technical level $(T)$, factor structure $(G)$, and policy regulations $(r)$ is negative, which means that $\mathrm{CO}_{2}$ emissions will be reduced due to the improvement of technology, factor structure and policy regulations; and it is expected that the increase of the GVC participating level will also have a negative effect on $\mathrm{CO}_{2}$ emissions.

\section{Methodology and data}

\section{GVC production length decomposition model}

According to the calculation method of Zhi et al. (2017a), this article will track the destinations and sources of value-added, and analyze GVC participation from forward and backward GVC production lengths. The process is as follows:

Divide the world into three parts: country $A$, country $B$ and other countries $(R)$. Each country has two industrial sectors: Industry 1 and Industry 2 . Then, the world input-output table will be reflected in Table 1.

Table 1 World input-output

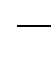

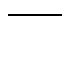

Intermediate Use B
Final Demand

A $\quad$ B $\quad$ R Output 
table

\begin{tabular}{|c|c|c|c|c|c|c|c|c|c|c|c|}
\hline & & & & & & & & & & & \\
\hline Country & Industry & 1 & 2 & 1 & 2 & 1 & 2 & & & & \\
\hline & 1 & $Z_{11}^{A A}$ & $Z_{12}^{A A}$ & $Z_{11}^{A B}$ & $Z_{12}^{A B}$ & $Z_{11}^{A R}$ & $Z_{12}^{A R}$ & $Y_{1}^{A A}$ & $Y_{1}^{A B}$ & $Y_{1}^{A R}$ & $X_{1}^{A}$ \\
\hline & 2 & $Z_{21}^{A A}$ & $Z_{22}^{A A}$ & $Z_{21}^{A B}$ & $Z_{22}^{A B}$ & $Z_{21}^{A R}$ & $Z_{22}^{A R}$ & $Y_{2}^{A A}$ & $Y_{2}^{A B}$ & $Y_{2}^{A R}$ & $X_{2}^{A}$ \\
\hline & 1 & $Z_{11}^{B A}$ & $Z_{12}^{B A}$ & $Z_{11}^{B B}$ & $Z_{12}^{B B}$ & $Z_{11}^{B R}$ & $Z_{12}^{B R}$ & $Y_{1}^{B A}$ & $Y_{1}^{B B}$ & $Y_{1}^{B R}$ & $X_{1}^{B}$ \\
\hline & 2 & $Z_{21}^{B A}$ & $Z_{22}^{B A}$ & $Z_{21}^{B B}$ & $Z_{22}^{B B}$ & $Z_{21}^{B R}$ & $Z_{22}^{B R}$ & $Y_{2}^{B A}$ & $Y_{2}^{B B}$ & $Y_{2}^{B R}$ & $X_{2}^{B}$ \\
\hline & 1 & $Z_{11}^{R A}$ & $Z_{12}^{R A}$ & $Z_{11}^{R B}$ & $Z_{12}^{R B}$ & $Z_{11}^{R R}$ & $Z_{12}^{R R}$ & $Y_{1}^{R A}$ & $Y_{1}^{R B}$ & $Y_{1}^{R R}$ & $X_{1}^{R}$ \\
\hline II & 2 & $Z_{21}^{R A}$ & $Z_{22}^{R A}$ & $Z_{21}^{R B}$ & $Z_{22}^{R B}$ & $Z_{21}^{R R}$ & $Z_{22}^{R R}$ & $Y_{2}^{R A}$ & $Y_{2}^{R B}$ & $Y_{2}^{R R}$ & $X_{2}^{R}$ \\
\hline Value & added & $V a_{1}^{A}$ & $V a_{2}^{A}$ & $V a_{1}^{B}$ & $V a_{1}^{B}$ & $V a_{1}^{R}$ & $V a_{2}^{R}$ & & & & \\
\hline Tot: & nput & $\left(X_{1}^{A}\right)^{\prime}$ & $\left(X_{2}^{A}\right)^{\prime}$ & $\left(X_{1}^{B}\right)^{\prime}$ & $\left(X_{2}^{B}\right)^{\prime}$ & $\left(X_{1}^{R}\right)^{\prime}$ & $\left(X_{2}^{R}\right)^{\prime}$ & & & & \\
\hline
\end{tabular}

212

Matrix $Z$ represents the intermediate inputs produced in one country and used in another country; vector $Y$ represents the final product produced in one country and used in another country; vector $X$ represents the total output of one country; vector Va represents one Country's direct value added.

Suppose the input coefficient matrix is $A=Z \hat{X}^{-1}, \hat{X}$ represents the diagonal matrix of $X$, at this time, $V=V a \hat{X}^{-1}$. And the total output $X$ can be expressed as:

$$
X=A X+Y=A^{D} X+Y^{D}+A^{F} X+Y^{F}=A^{D} X+Y^{D}+E
$$

$Y$ represents the sum of final products used in a country from other countries, $A^{D}$ represents the domestic input coefficient, $Y^{D}$ represents the total domestic final products consumed by each country, $A^{F}$ represents the import input coefficient, $Y^{F}$ represents the sum of final products exported, and $E$ represents total exports. According to the Leontief inverse matrix $(B)$, we can rewrite Equation(14):

$$
\begin{aligned}
& X=B Y=(I-A)^{-1} Y=\left(I-A^{D}\right)^{-1} Y^{D}+\left(I-A^{D}\right)^{-1} E \\
& =B^{D} Y^{D}+B^{D} E=B^{D} Y^{D}+B^{D} Y^{F}+B^{D} A^{F} X
\end{aligned}
$$

Among them, $B^{D}=\left(I-A^{D}\right)^{-1}$ represents the domestic Leontief inverse matrix. Based on this, the relationship between the value-added and the final product in Table 1 is:

$$
V a^{\prime}=\hat{V} X=\hat{V} B Y
$$

It can be seen that the initial input (value-added) of an industry can only be absorbed by the final product of the same industry. Therefore, the equation for the production process involved in the value-added can be summarized as follows:

$$
\hat{V} \hat{Y}+\hat{V} A \hat{Y}+\hat{V} A A \hat{Y}+\cdots=\hat{V}(I+A+A A+\cdots) \hat{Y}=\hat{V}(I-A)^{-1} \hat{Y}=\hat{V} B \hat{Y}
$$

$\hat{V} B \hat{Y}$ matrix represents the sum of value-added in all production stages, each element of which represents the value-added from an industry in one country, and the value-added is directly or indirectly used by an industry in another country to produce final products.

Take the production length of each stage as the weight and add it up to get the total output of a specific industrial department, we obtain:

$$
\begin{aligned}
& \hat{V} \hat{Y}+2 \hat{V A} \hat{Y}+3 \hat{V} A A \hat{Y}+\cdots=\hat{V}(I+2 A+3 A A+\cdots) \hat{Y} \\
& =\hat{V}(B+A B+A A B+\cdots)^{-1} \hat{Y}=\hat{V} B B \hat{Y}
\end{aligned}
$$

Therefore, the average production length of the value-added in the final product is: 


$$
P L v y=\frac{\hat{V} B B \hat{Y}}{\hat{V} B \hat{Y}}
$$

The average production length based on the forward industry linkage is:

$$
P L v=\frac{\hat{V} B B Y}{\hat{V} B Y}=\frac{\hat{V} B X}{\hat{V X}}
$$

Equation (20) measures the amount of supplementary value-added per unit of industry once, in which the value-added of each industry can be seen as a whole. At this time, the longer the forward production length is, the more downstream production stages the value-added participates in as a substitute, and the higher its upstream production position is.

The production length based on the backward industry linkage is:

$$
P L y=\frac{V B B \hat{Y}}{V B \hat{Y}}
$$

Equation (21) measures the total value-added input of final product in a specific industry. At this time, the longer the backward production length is, the more upstream production stages of a particular final product has, the lower the downstream production position of the product is.

According to the decomposition framework of value-added and final products proposed by Zhi et al. (2017b), the production activities of a country can be broken down into 5 parts according to the different situation of cross-border production activities:

$$
\begin{aligned}
& \hat{V} B \hat{Y}=\hat{V} B^{D} \hat{Y}^{D}+\hat{V} B^{D} \hat{Y}^{F}+\hat{V} B^{D} A^{F} B \hat{Y} \\
& =\underbrace{\hat{V} B^{D} \hat{Y}^{D}}_{(1)-V_{-} D}+\underbrace{\hat{V} B^{D} \hat{Y}^{F}}_{(2)-V_{-} R T}+\underbrace{\hat{V} B^{D} A^{F} B^{D} \hat{Y}^{D}}_{(3 a)-V_{-} G V C_{-} S}+\underbrace{\hat{V} B^{D} A^{F}\left(B \hat{Y}-B^{D} \hat{Y}^{D}\right)}_{(3 b)-V_{-} G V C_{-} C} \\
& =\underbrace{\hat{V} B^{D} \hat{Y}^{D}}_{(1)-V_{-} D}+\underbrace{\hat{V} B^{D} \hat{Y}^{F}}_{(2)-V_{-} R T}+\underbrace{\hat{V} B^{D} A^{F} B^{D} \hat{Y}^{D}}_{(3 a)-V_{-} G V C_{-} S}+\underbrace{\hat{V} B^{D}\left(A^{F} B\right)^{D} \hat{Y}}_{(4 a)-V_{-} G V C_{-} D}+\underbrace{\hat{V} B^{D}\left[\left(A^{F} B\right)^{F} \hat{Y}-A^{F} B^{D} \hat{Y}^{D}\right]}_{(4 b)-V_{-} G V C_{-} F}
\end{aligned}
$$

In Equation (22), (1)The first part is the domestic value-added, which refers to the part of domestically produced goods that are ultimately consumed domestically, represented by $V \_D$. (2) Part 2 represents the Ricardo trade part, that is, the final products exporting to foreign countries are directly consumed, which is only cross-border once, denoted by $V_{-} R T$. (3)The part representing cross-border production activities is divided into simple cross-border production activities and complex cross-border production activities. Simple cross-border production activity refers to the part of the intermediate product produced in one country and directly used by the importing country for production and consumption, the production activity is only cross-border once, denoted as $V_{-} G V C \_S$. Complex cross-border production activity refers to the part of intermediate goods produced in one country that are used by the importing country for production and exported to a third country, denoted as $V_{-} G V C_{-} C$. (4) The complex cross-border production activities involve two categories according to whether they return to the exporting country. Among them, $4 \mathrm{a}$ is the part returned to the exporting country and absorbed by the exporting country, denoted by $V_{-} G V C_{-} D .4 \mathrm{~b}$ is the part that is indirectly absorbed by the importing country and exported to other trading partner countries after being processed, denoted by $V \_G V C_{-} F$.

According to this, the GVC production length in Equation 19 is divided into five parts: 


$$
\begin{aligned}
& P L v y=\underbrace{\frac{\hat{V} B^{D} B^{D} \hat{Y}^{D}}{\hat{V} B^{D} \hat{Y}^{D}}}_{(1)-P L_{-} D}+\underbrace{\frac{\hat{V} B^{D} B^{D} \hat{Y}^{F}}{\hat{V} B^{D} \hat{Y}^{F}}}_{(2)-P L_{-} R T}+\underbrace{\frac{\hat{V} B B \hat{Y}-\hat{V} B^{D} B^{D} \hat{Y}^{D}}{\hat{V} B^{D} A^{F} B \hat{Y}}}_{(3)-P L_{-} G V C} \\
& =\underbrace{\frac{\hat{V} B^{D} B^{D} \hat{Y}^{D}}{\hat{V} B^{D} \hat{Y}^{D}}}_{(1)-P L_{-} D}+\underbrace{\frac{\hat{V} B^{D} B^{D} \hat{Y}^{F}}{\hat{V} B^{D} \hat{Y}^{F}}}_{(2)-P L_{-} R T}+\underbrace{\frac{\hat{V} B^{D} B^{D} A^{F} B^{D} \hat{Y}^{D}+\hat{V} B^{D} A^{F} B^{D} B^{D} \hat{Y}^{D}}{\hat{V} B^{D} A^{F} B \hat{Y}}}_{(3 a)-P L_{-} G V C_{-} S} \\
& +\underbrace{\frac{\hat{V} B^{D} B^{D} A^{F}\left(B \hat{Y}-B^{D} \hat{Y}^{D}\right)}{\hat{V} B^{D} A^{F}\left(B \hat{Y}-B^{D} \hat{Y}^{D}\right)}}_{(4 a)-P L_{-} G V C_{-} D}+\underbrace{\frac{\hat{V} B^{D} A^{F}\left(B B \hat{Y}-B^{D} B^{D} \hat{Y}^{D}\right)}{\hat{V} B^{D} A^{F}\left(B \hat{Y}-B^{D} \hat{Y}^{D}\right)}}_{(4 b)-P L_{-} G V C_{-} F}
\end{aligned}
$$

Similarly, as shown in Equations 24 and 25, the forward production length $(P L v)$ and the backward production length $(P L y)$ can also be divided into five parts. Among them, the part that participates in GVC activities include the GVC production length ( $\left.P L v_{-} G V C, P L y+G V C\right)$, the simple GVC production length ( $P L v_{-} G V C \_S, P L y_{-} G V C_{-} S$ ), the GVC production length returning to the exporting country $\left(P L v_{-} G V C_{-} D, P L y_{-} G V C_{-} D\right)$ and the pure foreign GVC production length ( $P L v_{-} G V C_{-} F$, PLy_GVC_F). The decomposition model of production length is shown in Fig. 1.

$$
\begin{aligned}
& P L v=\underbrace{\frac{\hat{V} B^{D} B^{D} Y^{D}}{\hat{V} B^{D} Y^{D}}}_{(1)-P L v_{-} D}+\underbrace{\frac{\hat{V} B^{D} B^{D} Y^{F}}{\hat{V} B^{D} Y^{F}}}_{(2)-P L v_{-} R T}+\underbrace{\frac{\hat{V} B B Y-\hat{V} B^{D} B^{D} Y^{D}}{\hat{V} B^{D} A^{F} B Y}}_{(3)-P L v_{-} G V C} \\
& =\underbrace{\frac{\hat{V} B^{D} B^{D} Y^{D}}{\hat{V} B^{D} Y^{D}}}_{(1)-P L v_{-} D}+\underbrace{\frac{\hat{V} B^{D} B^{D} Y^{F}}{\hat{V} B^{D} Y^{F}}}_{(2)-P L v_{-} R T}+\underbrace{\frac{\hat{V} B^{D} B^{D} A^{F} B^{D} Y^{D}+\hat{V} B^{D} A^{F} B^{D} B^{D} Y^{D}}{\hat{V} B^{D} A^{F} B Y}}_{(3 a)-P L v_{-} G V C_{-} S} \\
& +\underbrace{\frac{\hat{V} B^{D} B^{D} A^{F}\left(B Y-B^{D} Y^{D}\right)}{\hat{V} B^{D} A^{F}\left(B Y-B^{D} Y^{D}\right)}}_{(4 a)-P L v_{-} G V C_{-} D}+\underbrace{\frac{\hat{V} B^{D} A^{F}\left(B B Y-B^{D} B^{D} Y^{D}\right)}{\hat{V} B^{D} A^{F}\left(B Y-B^{D} Y^{D}\right)}}_{(4 b)-P L v_{-} G V C_{-} F} \\
& P L y=\underbrace{\frac{V B^{D} B^{D} \hat{Y}^{D}}{V B^{D} \hat{Y}^{D}}}_{(1)-P L y_{-} D}+\underbrace{\frac{V B^{D} B^{D} \hat{Y}^{F}}{V B^{D} \hat{Y}^{F}}}_{(2)-P L y_{-} R T}+\underbrace{\frac{V B B \hat{Y}-V B^{D} B^{D} \hat{Y}^{D}}{V B^{D} A^{F} B \hat{Y}}}_{(3)-P L y_{-} G V C} \\
& =\underbrace{\frac{V B^{D} B^{D} \hat{Y}^{D}}{V B^{D} \hat{Y}^{D}}}_{(1)-P L_{y_{-} D}}+\underbrace{\frac{V B^{D} B^{D} \hat{Y}^{F}}{V B^{D} \hat{Y}^{F}}}_{(2)-P L y_{-} R T}+\underbrace{\frac{V B^{D} B^{D} A^{F} B^{D} \hat{Y}^{D}+V B^{D} A^{F} B^{D} B^{D} \hat{Y}^{D}}{V B^{D} A^{F} B \hat{Y}}}_{(3 a)-P L_{y_{-}} G V C_{-} S} \\
& +\underbrace{\frac{V B^{D} B^{D} A^{F}\left(B \hat{Y}-B^{D} \hat{Y}^{D}\right)}{V B^{D} A^{F}\left(B \hat{Y}-B^{D} \hat{Y}^{D}\right)}}_{(4 a)-P L y_{-} G V C_{-} D}+\underbrace{\frac{V B^{D} A^{F}\left(B B \hat{Y}-B^{D} B^{D} \hat{Y}^{D}\right)}{V B^{D} A^{F}\left(B \hat{Y}-B^{D} \hat{Y}^{D}\right)}}_{(4 b)-P L y_{-} G V C_{-} F}
\end{aligned}
$$




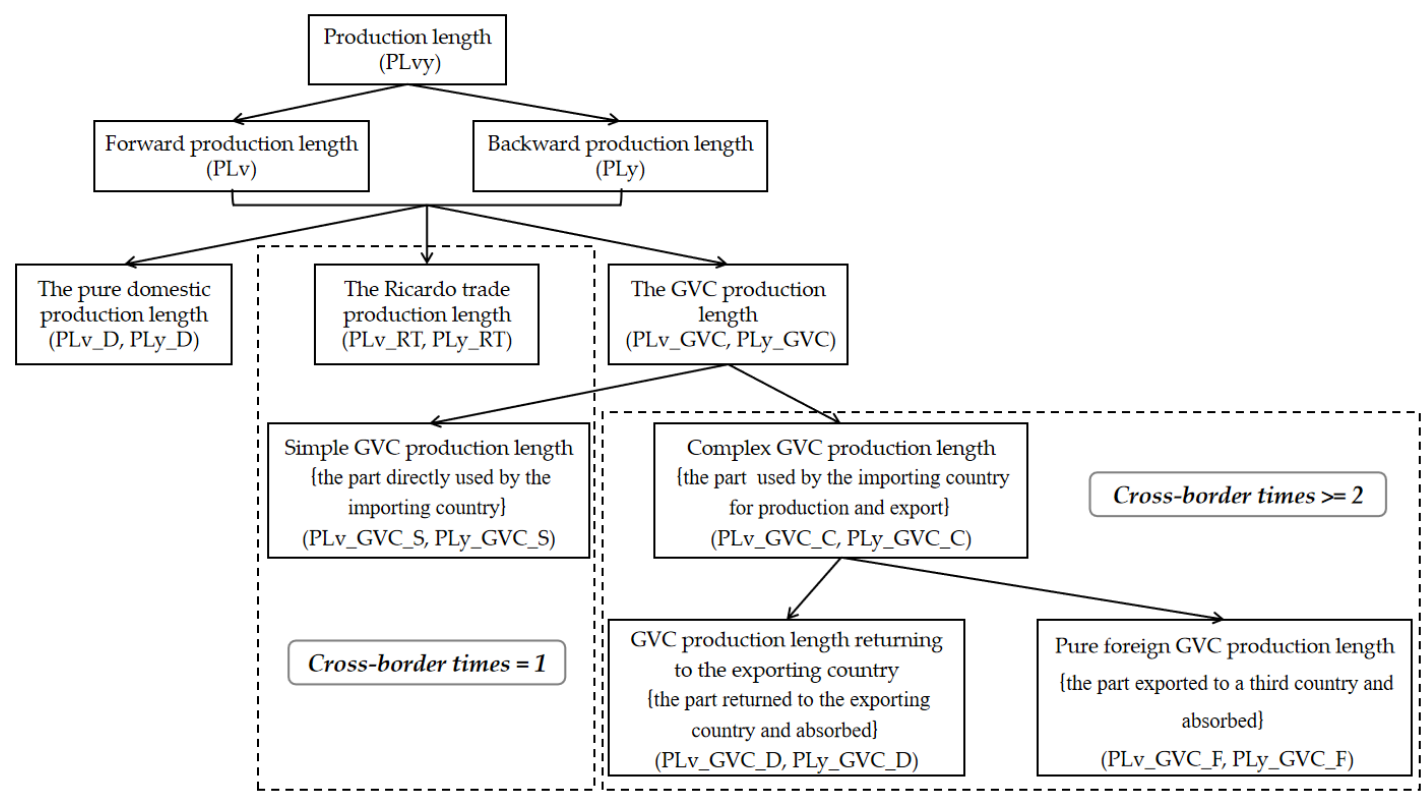

Fig. 1 The decomposition model of production length

\section{STIRPAT model}

In order to test the real impact of the indicators in the theoretical model, this part studies the specific impact of GVC production length on $\mathrm{CO}_{2}$ emissions of China's equipment manufacturing industry by constructing a STIRPAT (Stochastic Impacts by Regression on PAT) model. The prototype of the STIRPAT model is the IPAT model, which was first proposed by Enrlich and Holden, and has been widely used in the field of environmental contamination research (Hofmann et al. 2016). In the IPAT model, the environmental pressure $(I)$ is determined by the population size $(P)$, per capita assets $(A)$, and technology level $(T)$. Its general form is as follows:

$$
I=P A T
$$

As shown in Equation (26), the IPAT model reflects the impact of population growth and other factors on environmental pressure. However, when the IPAT model describes the relationship between environmental impacts and various driving factors, it can only reflect changes in the same proportion, limiting other possible impact results, and the model cannot perform hypothesis testing, so York and Dietz proposed the STIRPAT model, which is:

$$
I_{i}=a P_{i}^{b} A_{i}^{c} T_{i}^{d} e_{i}
$$

Take the logarithm of both sides of the Equation to rewrite Equation (27) into the additive mode:

$$
\ln I_{i}=a+b \ln P_{i}+c \ln A_{i}+d \ln T_{i}+e_{i}
$$

The STIRPAT model converts the IPAT statistical model to an ordinary linear model, which can be hypothesized tested by statistical methods, and the different impact strength of each impact factor can be estimated. The independent variables $P, A$ and $T$ can be replaced with other variables related to the main research object ( $\mathrm{Li} 2019$ ). Therefore, based on the classic STIRPAT model and the actual situation of the impact of participating in $\mathrm{GVC}$ on $\mathrm{CO}_{2}$ emissions, this paper improves and replaces some of the influencing factors, and finally builds the following empirical model: 


$$
\begin{aligned}
& \ln C_{i t}=\beta_{0}+\beta_{1} \ln V_{i t}+\beta_{2} \ln P_{\text {olicy }}+\beta_{3} \ln \text { Scale }_{i t}+\beta_{4} \ln G_{-} \text {factor }_{t} \\
& +\beta_{5} \ln T_{-} f d_{i t}+\beta_{6} \ln T_{-} \text {Rdit }_{i t}+\varepsilon_{i t}
\end{aligned}
$$

In Equation (29), the explained variable $C$ represents the $\mathrm{CO}_{2}$ emissions of each sub-industry of China's equipment manufacturing industry, which is a substitute for the environmental pressure in the IPAT model; $V$ represents the core explanatory variables related to the GVC, including forward and backward GVC production length $\left(P L v_{-} G V C, P L y_{-} G V C\right)$ and its decomposed parts; Policy represents the policy regulation, which is expressed by the amount of industrial pollution control investment based on the method of Peng and Li (2013), and the weight is the ratio of the total investment in fixed assets of equipment manufacturing industry to China's total investment in fixed assets, then the industrial pollution control investment is calculated according to China's total pollution control investment; Scale represents the production scale, instead of the population size in the original IPAT model, it is measured by per capita output value; G_factor is the factor structure, replacing the per capita assets in the original IPAT model, expressed by the ratio of the total fixed assets of industrial enterprises above designated size to the average number of industrial employees in each industry; in this article, the technical level in the original IPAT model is jointly replaced by $T_{-} f d i$ and $T_{-} R d$, and using the method of $\mathrm{Xu}$ (2019), foreign direct investment ( $\left.T_{-} f d i\right)$ is measured by the proportion of total assets of Hong Kong, Macao, Taiwan and foreign-invested industrial enterprises in total assets of all industrial enterprises above designated size, and R\&D investment $\left(T_{-} R d\right)$ measured by the R\&D expenditures of various industries; $\beta_{0}$ represents a constant term; $\beta_{1}$ to $\beta_{7}$ represent the coefficient of each variables, $\varepsilon$ represents a random disturbance term, $i$ represents an industry, and $t$ represents time.

\section{Data Sources}

Based on the International Standard Industrial Classification (ISIC Rev.4) and China's National Economic Standard Industrial Classification (GB/4757-2002), this article merges China's equipment manufacturing industry into five sub-industries, and selects the sample period from 2000 to 2014. Among them, the $\mathrm{CO}_{2}$ emission data is the original data of the latest environmental account released by the WIOD database in 2019; the data of the forward GVC production length, the backward GVC production length and the value-added of each industry are all calculated by the input-output account released by WIOD in 2016; R\&D expenditure data comes from the "Statistical Yearbook of Scientific and Technological Activities of Industrial Enterprises"; the rest of the data all comes from the "China Statistical Yearbook".

\section{Empirical results}

\section{The GVC production length of China's equipment manufacturing industry}

The results of the forward and backward GVC production length of China's equipment manufacturing industry is shown in Fig. 2 and Fig. 3. China's equipment manufacturing industry has become deeply participating in GVC, and the change trend of forward and backward GVC production length is similar, but the backward GVC production length is always longer than the forward GVC production length. In 2001, the production length of the forward and backward GVC production length increased rapidly, which thanks to the tremendous progress that China made after joining the WTO in 2000, the convenience of participating in the international division of production has been promoted, and the technology spillovers from developed countries has increased. After a difficult growth process, the 
Fig.3 The backward GVC production length of China's equipment manufacturing industry

Fig.2 The forward GVC production length of China's equipment manufacturing industry
GVC participating level of China's equipment manufacturing industry achieved a major leap again in 2009. Comparing the GVC production length under different participating modes, the largest increase part is the GVC production length returning to the exporting country, followed by the pure foreign GVC production length. The variation of the GVC production length and the simple GVC production length is almost the same. It shows that the impetus provided by participating in GVC is far greater than that of China's independent research and development.
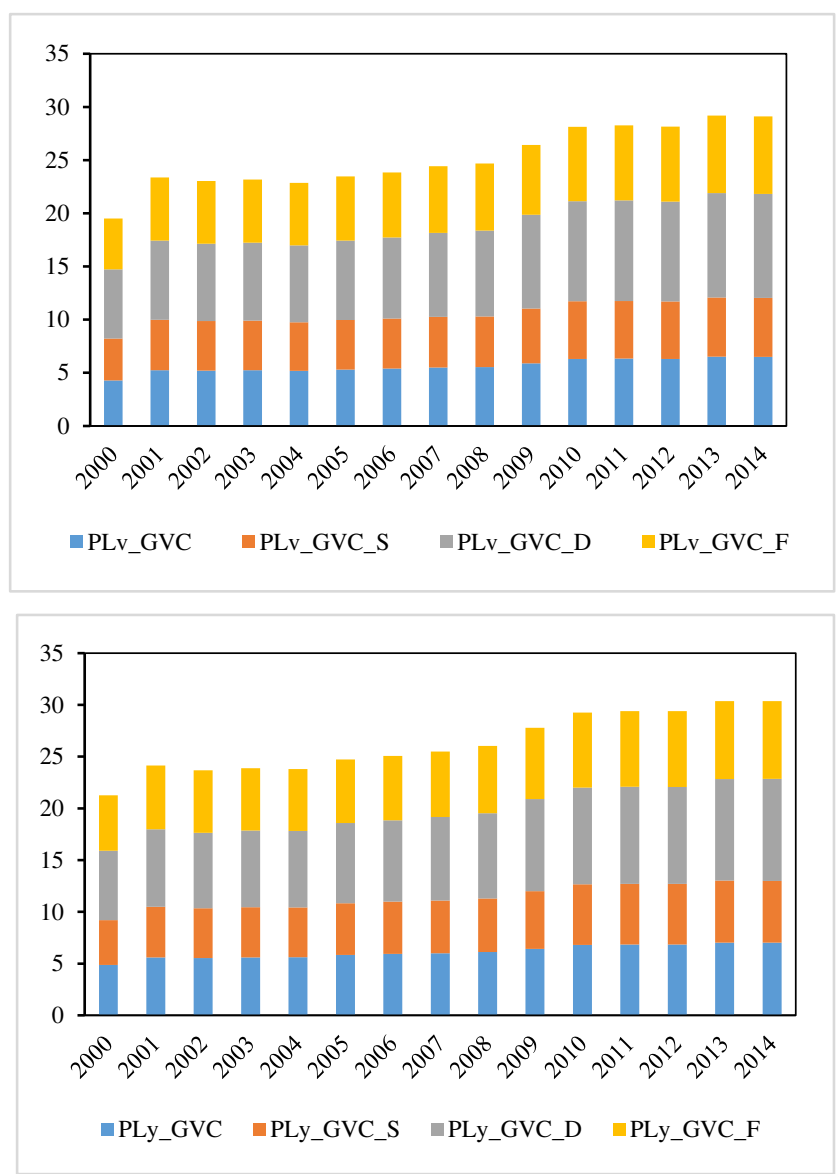

\section{$\mathrm{CO}_{2}$ emissions of China's equipment manufacturing industry}

The $\mathrm{CO}_{2}$ emissions of China's equipment manufacturing industry show obvious characteristics of industry clusters and stage distribution (Fig. 4). At first, the characteristics of industry clusters of $\mathrm{CO}_{2}$ emissions mainly reflect in the transportation equipment manufacturing industry. The $\mathrm{CO}_{2}$ emissions of the transportation equipment manufacturing industry accounted for about $73.19 \%$ of the total $\mathrm{CO}_{2}$ emissions of the equipment manufacturing industry. The $\mathrm{CO}_{2}$ emissions in 2014 reached 6.918 million tons, which is the main reason for the significant increase of the $\mathrm{CO}_{2}$ emissions of the equipment manufacturing industry. The machinery and equipment manufacturing industry ranked second, and its $\mathrm{CO}_{2}$ emissions increased rapidly in 2010, with an average annual proportion of about $15.76 \%$; computer, electronic and optical product manufacturing ranked third, with an average annual proportion of $7.92 \%$; fabricated metal products industry and electrical equipment manufacturing industry followed closely, with an average annual proportion of $2.43 \%$ and $0.7 \%$, respectively. In contrast, the $\mathrm{CO}_{2}$ emissions of the transportation equipment manufacturing industry far exceed those of 
times than that of high-tech industries. Meanwhile, the $\mathrm{CO}_{2}$ emission trend of China's equipment manufacturing industry is mainly divided into three stages. The first stage is the slow growth stage from 2000 to 2003. In this stage, the $\mathrm{CO}_{2}$ emissions of various industries are slowly increasing, and the annual growth rate is maintained at a relatively stable level. The total $\mathrm{CO}_{2}$ emissions in 2003 is about 392.98 million tons. The second stage was from 2004 to 2011. Thanks to the strong support of the Chinese government for the equipment manufacturing industry, the output value of the equipment manufacturing industry in this stage increased rapidly, resulting in a substantial increase in $\mathrm{CO}_{2}$ emissions, which reached a peak in 2011 at approximately 927.00 million tons. The third stage is the period of fluctuating growth from 2012 to 2014. But except for the $\mathrm{CO}_{2}$ emission growth rate of the fabricated metal products industry which rebounded in 2014, the $\mathrm{CO}_{2}$ emission growth rate of other sub-sectors is decreasing. The $\mathrm{CO}_{2}$ emissions of computer, electronic and optical products manufacturing industry and electrical equipment manufacturing industry also showed negative growth, indicating that China's equipment manufacturing industry has good prospects for $\mathrm{CO}_{2}$ reduction.

Fig. 4 The $\mathrm{CO}_{2}$ emission of China's equipment manufacturing industry from 2000 to 2014

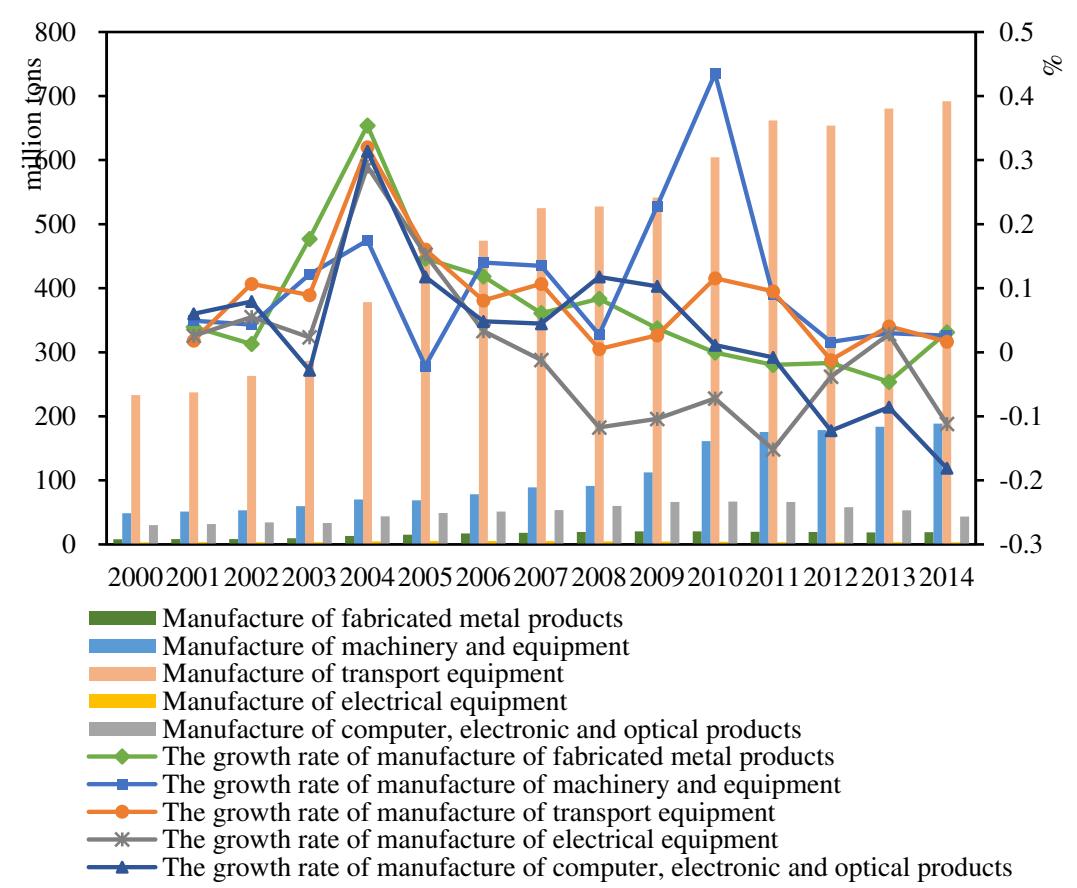

\section{The impact of the GVC production length on $\mathrm{CO}_{2}$ emissions}

This paper uses the panel data of China's equipment manufacturing industry from 2000 to 2014 to perform regression analysis on Equation (31). At first, in order to avoid endogenous problems, we applied the LLC test and ADF-Fisher test, the outcomes indicates that the panel data sets follow the stationary process (Table 2 ).

Table 2 Panel unit root test results

\begin{tabular}{lccccc}
\hline \multirow{2}{*}{ Variables } & LLC & ADF & \multirow{2}{*}{ Variables } & LLC & ADF \\
& Test & Test & & Test \\
\hline
\end{tabular}




\begin{tabular}{|c|c|c|c|c|c|}
\hline \multirow[b]{2}{*}{ C } & -1.7440 & -2.7329 & \multirow{2}{*}{ PLv_GVC_S } & -4.1954 & -2.9046 \\
\hline & $(0.0406)$ & $(0.0031)$ & & $(0.0000)$ & $(0.0018)$ \\
\hline \multirow{2}{*}{ Policy } & -4.6917 & -4.3130 & \multirow{2}{*}{ PLv_GVC_D } & -4.8818 & -5.6982 \\
\hline & $(0.0000)$ & $(0.0000)$ & & $(0.0000)$ & $(0.0000)$ \\
\hline \multirow{2}{*}{ Scale } & -4.5352 & -3.8243 & \multirow{2}{*}{ PLv_GVC_F } & -4.7051 & -5.3724 \\
\hline & $(0.0000)$ & $(0.0001)$ & & $(0.0000)$ & $(0.0000)$ \\
\hline \multirow{2}{*}{ G_factor } & -1.5983 & -2.5289 & \multirow{2}{*}{ PLy_GVC } & -4.6544 & -3.5775 \\
\hline & $(0.0550)$ & $(0.0057)$ & & $(0.0000)$ & $(0.0002)$ \\
\hline \multirow{2}{*}{ T_fdi } & -3.9857 & -2.4197 & \multirow{2}{*}{ PLy_GVC_S } & -4.2320 & -3.5288 \\
\hline & $(0.0000)$ & $(0.0078)$ & & $(0.0000)$ & $(0.0002)$ \\
\hline \multirow{2}{*}{ T_Rd } & -2.0939 & -2.3833 & \multirow{2}{*}{ PLy_GVC_D } & -9.5204 & -6.6370 \\
\hline & $(0.0181)$ & $(0.0086)$ & & $(0.0000)$ & $(0.0000)$ \\
\hline \multirow{2}{*}{ PLv_GVC } & -4.0849 & -4.3532 & \multirow{2}{*}{ PLy_GVC_F } & -5.4928 & -6.4258 \\
\hline & $(0.0000)$ & $(0.0000)$ & & $(0.0000)$ & $(0.0000)$ \\
\hline
\end{tabular}

372

373

Then, according to the results of the F test of the panel data, the fixed effects model is better than the mixed regression model. Finally, because the original hypothesis of the Hausman test is that there is a random effect, and the results of the Hausman test in this article reject the null hypothesis, indicating that the fixed effect model is better than the random effect model. Based on this, the empirical estimation results are as follows.

The first three columns in Table 3 are the regression results with only control variables added. The results show that policy regulation, industrial scale, factor structure, foreign direct investment and $\mathrm{R} \& \mathrm{D}$ investment all have a significant impact on $\mathrm{CO}_{2}$ emissions. However, the coefficient of determination $\mathrm{R}^{2}$ in column (1) is only 0.558 , which is much lower than 0.757 in column (2), indicating that $19.9 \%$ of $\mathrm{CO}_{2}$ emissions changes are caused by individual differences that do not change over time. The fixed effect model is more accurate in estimating the indicators in this article. After continuing to add annual dummy variables in column (3), the impact of policy regulation and foreign investment on $\mathrm{CO}_{2}$ emissions has become significant, and the coefficient of determination $\mathrm{R}^{2}$ has increased to 0.853 , indicating a $9.6 \%$ change in $\mathrm{CO}_{2}$ emissions of the equipment manufacturing industry can be explained by missing variables that change with time but not with industry.

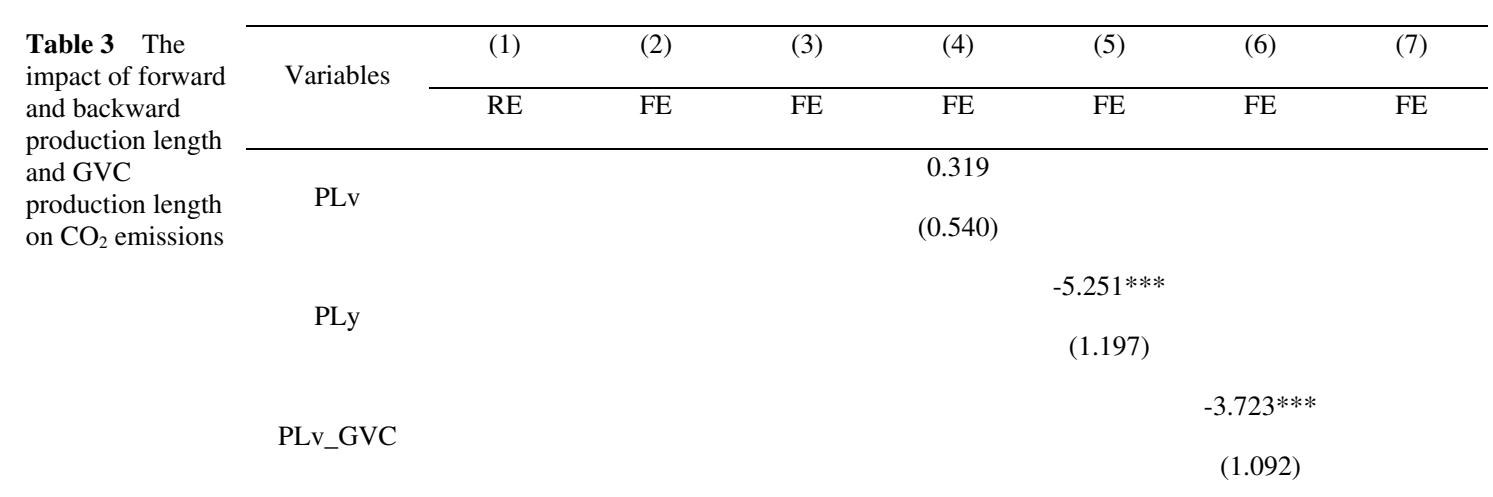




\begin{tabular}{|c|c|c|c|c|c|c|c|}
\hline \multicolumn{7}{|l|}{ PLy_GVC } & $\begin{array}{c}-5.466^{* * *} \\
(1.658)\end{array}$ \\
\hline \multirow{2}{*}{ Policy } & $1.056^{* * *}$ & -0.0747 & $-0.394 * * *$ & $-0.389^{* * *}$ & $-0.273^{* *}$ & $-0.483^{* * *}$ & $-0.244 * *$ \\
\hline & $(0.324)$ & $(0.0742)$ & $(0.117)$ & $(0.118)$ & $(0.104)$ & $(0.109)$ & $(0.116)$ \\
\hline \multirow{2}{*}{ Scale } & $-5.490 * * *$ & $0.956^{* * *}$ & $2.139 * * *$ & $2.128 * * *$ & $1.016^{*}$ & $2.541 * * *$ & 1.087 \\
\hline & (1.395) & $(0.297)$ & $(0.623)$ & $(0.628)$ & $(0.593)$ & $(0.579)$ & $(0.654)$ \\
\hline \multirow{2}{*}{ G_factor } & $6.223 * * *$ & $-1.348 * * *$ & $-3.088 * * *$ & $-3.081 * * *$ & $-1.609^{* *}$ & $-3.653^{* * *}$ & $-2.192 * * *$ \\
\hline & $(1.431)$ & $(0.398)$ & $(0.785)$ & $(0.790)$ & $(0.753)$ & $(0.733)$ & $(0.768)$ \\
\hline \multirow{2}{*}{ T_fdi } & $1.886^{* * *}$ & 0.0890 & $-0.885^{* * *}$ & $-0.919 * * *$ & -0.288 & $-1.196 * * *$ & -0.348 \\
\hline & $(0.504)$ & (0.193) & $(0.255)$ & $(0.263)$ & $(0.258)$ & $(0.249)$ & $(0.285)$ \\
\hline \multirow{2}{*}{ T_Rd } & -0.0304 & $0.483^{* * * *}$ & $1.440 * * *$ & $1.477 * * *$ & $1.155^{* * * *}$ & $1.385^{* * *}$ & $1.290 * * *$ \\
\hline & $(0.187)$ & $(0.177)$ & $(0.254)$ & $(0.262)$ & $(0.227)$ & $(0.231)$ & $(0.237)$ \\
\hline \multirow{2}{*}{ _cons } & $15.95^{* * *}$ & 1.240 & -2.052 & -2.413 & $7.006^{* * * *}$ & 2.556 & $9.589 * *$ \\
\hline & $(3.371)$ & $(0.773)$ & (1.396) & (1.532) & $(2.387)$ & (1.855) & (3.755) \\
\hline Industry FE & YES & YES & YES & YES & YES & YES & YES \\
\hline Year FE & NO & NO & YES & YES & YES & YES & YES \\
\hline R-Squared & 0.558 & 0.757 & 0.853 & 0.854 & 0.894 & 0.881 & 0.879 \\
\hline $\begin{array}{c}\text { Hausman } \\
\text { Test }\end{array}$ & \multicolumn{2}{|c|}{$67.26 / 0.0000$} & & & & & \\
\hline $\mathrm{N}$ & 75 & 75 & 75 & 75 & 75 & 75 & 75 \\
\hline
\end{tabular}

Note: The robust standard errors in parentheses, ***, **, and * indicate significant at the level of $1 \%, 5 \%$, and $10 \%$ respectively.

With the acceleration of globalization, the impact of participating in $\mathrm{GVC}$ on $\mathrm{CO}_{2}$ emissions cannot be ignored. Since production length variables change with time and individual differences, columns (4) to (7) in Table 3 is based on column (3) and added variables reflecting the production length of China's equipment manufacturing industry to test the specific impact of GVC production length on $\mathrm{CO}_{2}$ emissions. Except for the forward production length, the impact of the backward production length, the forward GVC production length and the backward GVC production length on the $\mathrm{CO}_{2}$ emissions of the equipment manufacturing industry have all passed the test at a significant level of $1 \%$, and the coefficients of the three are all negative, which means that for every $1 \%$ increase in the backward production length, the forward GVC production length and the backward GVC production length, the level of $\mathrm{CO}_{2}$ emissions will drop by about $5.3 \%, 3.7 \%$, and $5.5 \%$, respectively. The $\mathrm{CO}_{2}$ emission reduction effect of the GVC is obvious, which further supports the derivation in the previous model. Column (6) shows that the extension of the forward GVC production length can effectively reduce $\mathrm{CO}_{2}$ emission, indicating that the equipment manufacturing industry has achieved technological improvement through imitation, learning and secondary innovation in the process of moving upstream to the international division of production. But this reduction is still not enough to drive the overall transformation of the $\mathrm{CO}_{2}$ emissions of intermediate products in the equipment manufacturing industry, making the $\mathrm{CO}_{2}$ emission reduction effect from the perspective of forward production length not obvious, which also shows that the production technology of industrial intermediate products of China's domestic equipment manufacturing industry is still not environmentally friendly, and the improvement of clean technology in the domestic equipment manufacturing industry has stuck in a 
"bottleneck period". It can be seen from columns (5) and (7) that the $\mathrm{CO}_{2}$ emission reduction effect of the backward production length and the backward GVC production length is similar, both are greater than the result of the forward GVC production length. It proves that while the participation of China's equipment manufacturing industry in GVC activities has increased, the clean level of final product production technology has been greatly improved, and it has gradually moved from a low-tech, high- $\mathrm{CO}_{2}$ production stage to a high-tech, low- $\mathrm{CO}_{2}$ production stage.

According to the data in each column in Table 4, whether it is the forward GVC production length or the backward GVC production length, the extension of the simple GVC production length has the best reduction effect on $\mathrm{CO}_{2}$ emissions, both are significantly negative at the $1 \%$ level. However, the length of complex GVC production length has little effect on $\mathrm{CO}_{2}$ emissions, and only the backward pure foreign GVC production length has passed the test and has a positive effect on $\mathrm{CO}_{2}$ emissions. This means that only one of the three types of intermediate products exported by China's equipment manufacturing industry has played a huge role in promoting $\mathrm{CO}_{2}$ emission reduction. The first type is the intermediate products directly used and consumed by the importing country. The GVC production length is expressed as $P L v_{-} G V C_{-} S$ and $P L y_{-} G V C_{-} S$. The $\mathrm{CO}_{2}$ emissions of such products decrease with the extension of the production length, indicating that China's cleaner production technology for this type of product has been promoted. The second type is the intermediate product used by the importing country to produce and return to the exporting country. Its GVC production length is expressed as $P L v_{-} G V C_{-} D$ and $P L y_{-} G V C_{-} D$. The poor production performance of this type of product indicates that even if the final products that meet domestic needs also needs to be processed by foreign companies before it is used. It further points out the urgency of China's equipment manufacturing industry to improve the level of production technology. The third type is intermediate products that are used by importing countries for production and exported to third countries, expressed as $P L v_{-} G V C_{-} F$ and $P L y \_G V C_{-} F$. The extension of the production length of such products will promote the increase of $\mathrm{CO}_{2}$ emissions. The reason is that the participation level of international production of China's equipment manufacturing industry is not high, the industry mainly participates in the GVC through simple processing and production links. The processing technology of complex intermediate products is still immature, when China processes and assembles imported high value-added intermediate imports, it keeps a large amount of $\mathrm{CO}_{2}$ emissions in the country (Zhao and Yang 2020).

\begin{tabular}{|c|c|c|c|c|c|c|c|}
\hline \multirow{7}{*}{$\begin{array}{l}\text { Table } 4 \text { The impact of } \\
\text { the decomposition of the } \\
\mathrm{GVC}_{\text {production length }} \\
\text { on } \mathrm{CO}_{2} \text { emissions }\end{array}$} & \multirow{2}{*}{ Variables } & \multicolumn{3}{|c|}{ Forward GVC production length } & \multicolumn{3}{|c|}{ Backward GVC production length } \\
\hline & & (1) & (2) & (3) & (4) & $(5)$ & (6) \\
\hline & PLv_GVC_S & $\begin{array}{c}-2.922^{* * * *} \\
(0.985)\end{array}$ & & & & & \\
\hline & PLv_GVC_D & & $\begin{array}{l}-0.120 \\
(0.723)\end{array}$ & & & & \\
\hline & PLv_GVC_F & & & $\begin{array}{c}0.496 \\
(0.666)\end{array}$ & & & \\
\hline & PLy_GVC_S & & & & $\begin{array}{c}-3.923 * * * \\
(1.322)\end{array}$ & & \\
\hline & PLy_GVC_D & & & & & 1.127 & \\
\hline
\end{tabular}




\begin{tabular}{|c|c|c|c|c|c|c|}
\hline PLy_GVC_F & & & & & & $\begin{array}{l}1.320^{*} \\
(0.800)\end{array}$ \\
\hline Policy & $\begin{array}{c}-0.489 * * * \\
(0.113)\end{array}$ & $\begin{array}{c}-0.391 * * * \\
(0.119)\end{array}$ & $\begin{array}{c}-0.393 * * * \\
(0.117)\end{array}$ & $\begin{array}{c}-0.272 * * \\
(0.116)\end{array}$ & $\begin{array}{c}-0.419 * * * \\
(0.119)\end{array}$ & $\begin{array}{c}-0.402 * * * \\
(0.115)\end{array}$ \\
\hline Scale & $\begin{array}{c}2.463 * * * \\
(0.591)\end{array}$ & $\begin{array}{c}2.143 * * * \\
(0.630)\end{array}$ & $\begin{array}{c}2.064 * * * \\
(0.634)\end{array}$ & $\begin{array}{l}1.370^{* *} \\
(0.636)\end{array}$ & $\begin{array}{c}2.095 * * * \\
(0.624)\end{array}$ & $\begin{array}{c}2.084 * * * \\
(0.614)\end{array}$ \\
\hline G_factor & $\begin{array}{c}-3.501 * * * \\
(0.744)\end{array}$ & $\begin{array}{c}-3.103 * * * \\
(0.797)\end{array}$ & $\begin{array}{c}-2.981 * * * \\
(0.801)\end{array}$ & $\begin{array}{c}-2.429 * * * \\
(0.764)\end{array}$ & $\begin{array}{c}-2.994 * * * \\
(0.789)\end{array}$ & $\begin{array}{c}-3.027 * * * \\
(0.773)\end{array}$ \\
\hline T_fdi & $\begin{array}{c}-1.186 * * * \\
(0.258)\end{array}$ & $\begin{array}{c}-0.889 * * * \\
(0.259)\end{array}$ & $\begin{array}{c}-0.792 * * * \\
(0.285)\end{array}$ & $\begin{array}{l}-0.524 * \\
(0.267)\end{array}$ & $\begin{array}{c}-0.870 * * * \\
(0.255)\end{array}$ & $\begin{array}{c}-0.792 * * * \\
(0.257)\end{array}$ \\
\hline T_Rd & $\begin{array}{c}1.452 * * * \\
(0.236)\end{array}$ & $\begin{array}{c}1.448^{* * *} \\
(0.260)\end{array}$ & $\begin{array}{c}1.394 * * * \\
(0.262)\end{array}$ & $\begin{array}{c}1.365^{* * * *} \\
(0.237)\end{array}$ & $\begin{array}{c}1.432 * * * \\
(0.253)\end{array}$ & $\begin{array}{c}1.414 * * * \\
(0.250)\end{array}$ \\
\hline _cons & $\begin{array}{l}1.196 \\
(1.700)\end{array}$ & $\begin{array}{l}-1.811 \\
(2.022)\end{array}$ & $\begin{array}{l}-2.708 \\
(1.657)\end{array}$ & $\begin{array}{l}5.805^{*} \\
(2.950)\end{array}$ & $\begin{array}{l}-4.319 \\
(2.579)\end{array}$ & $\begin{array}{c}-8.783 * * * \\
(2.130)\end{array}$ \\
\hline Industry FE & YES & YES & YES & YES & YES & YES \\
\hline Year FE & YES & YES & YES & YES & YES & YES \\
\hline R-Squared & 0.875 & 0.853 & 0.854 & 0.875 & 0.856 & 0.860 \\
\hline $\mathrm{N}$ & 75 & 75 & 75 & 75 & 75 & 75 \\
\hline
\end{tabular}

Note: The robust standard errors in parentheses, ${ }^{* * *}, * *$, and $*$ indicate significant at the level of $1 \%, 5 \%$, and $10 \%$ respectively.

\section{The impact of the relevant economic indicators on $\mathrm{CO}_{2}$ emissions}

Observing the data in Tables 3 and Table 4, we can see that policy regulations, factor structure, and foreign investment have a negative impact on $\mathrm{CO}_{2}$ emissions, and scale effects and $\mathrm{R} \& \mathrm{D}$ investment will promote the increase of $\mathrm{CO}_{2}$ emissions. In addition to $\mathrm{R} \& \mathrm{D}$ investment, the effects of other indicators on $\mathrm{CO}_{2}$ emissions are in line with expected results. The specific analysis is as follows: (1) The coefficient of policy regulation is maintained at around -0.4 , and the promotion of $\mathrm{CO}_{2}$ emission reduction is not obvious. This is related to China's industrialization development stage during 2000-2011, and environmental regulation did not take effect until it is over. (2) The production scale has an increasing effect on $\mathrm{CO}_{2}$ emissions, because in the process of joining the international division of production, China's equipment manufacturing industry has undertaken the transfer of high- $\mathrm{CO}_{2}$ emission industries from developed countries, and production is mainly based on high-energy and high-polluting activities. The expansion of production scale will lead to an increase in $\mathrm{CO}_{2}$ emissions, which is consistent with the reality. (3) The factor structure has a restraining effect on $\mathrm{CO}_{2}$ emissions. The factor structure of China's equipment manufacturing industry is changing from labor-intensive to capital-intensive, and it is still in the process of moving towards technology-intensive. The prospects for reducing $\mathrm{CO}_{2}$ emissions through the adjustment of the factor structure are great. (4) There is a significant negative correlation between foreign investment and $\mathrm{CO}_{2}$ emissions, indicating that the clean technology learned from the investing country can inhibit $\mathrm{CO}_{2}$ emissions with the spillover effect of FDI technology. (5) The effect of R\&D investment on $\mathrm{CO}_{2}$ emissions is positive and insignificant, which is consistent with the results of Wang et al. (2015). The reason is that, on the one hand, because 
the current Chinese enterprises cannot effectively allocate R\&D resources, the actual investment in clean technology is much lower than expected; on the other hand, it is because the current level of $\mathrm{CO}_{2}$ emission reduction technology of the equipment manufacturing industry is extremely low.

\section{Robustness check}

For the purpose of further examine the robustness of the empirical results, this paper removes $5 \%$ of the extreme values from both ends, and performs regression test on the sub-samples to eliminate the influence of non-randomness on the regression results (Table 5). The sample results are almost as same as the benchmark regression results, indicating that the research conclusions have strong robustness.

Table 5 Robustness test

\begin{tabular}{|c|c|c|c|c|c|c|c|c|}
\hline \multirow{2}{*}{ Variables } & \multicolumn{4}{|c|}{ Forward GVC production length } & \multicolumn{4}{|c|}{ Backward GVC production length } \\
\hline & (1) & (2) & (3) & (4) & (5) & (6) & (7) & (8) \\
\hline PLv_GVC & $\begin{array}{c}-2.354 * * \\
(0.938)\end{array}$ & & & & & & & \\
\hline PLv_GVC_S & & $\begin{array}{c}-2.280 * * \\
(0.934)\end{array}$ & & & & & & \\
\hline PLv_GVC_D & & & $\begin{array}{l}-0.188 \\
(0.740)\end{array}$ & & & & & \\
\hline PLv_GVC_F & & & & $\begin{array}{l}0.304 \\
(0.649)\end{array}$ & & & & \\
\hline PLy_GVC & & & & & $\begin{array}{c}-2.795^{* *} \\
(1.405)\end{array}$ & & & \\
\hline PLy_GVC_S & & & & & & $\begin{array}{c}-2.594 * * \\
(1.131)\end{array}$ & & \\
\hline PLy_GVC_D & & & & & & & $\begin{array}{l}0.605 \\
(0.985)\end{array}$ & \\
\hline PLy_GVC_F & & & & & & & & $\begin{array}{l}1.079 * \\
(0.628)\end{array}$ \\
\hline Policy & $\begin{array}{c}-0.417 * * * \\
(0.111)\end{array}$ & $\begin{array}{c}-0.451^{* * *} \\
(0.114)\end{array}$ & $\begin{array}{c}-0.389 * * * \\
(0.119)\end{array}$ & $\begin{array}{c}-0.395 * * * \\
(0.117)\end{array}$ & $\begin{array}{c}-0.277 * * \\
(0.128)\end{array}$ & $\begin{array}{c}-0.273^{* *} \\
(0.124)\end{array}$ & $\begin{array}{c}-0.408 * * * \\
(0.119)\end{array}$ & $\begin{array}{c}-0.407^{* * *} \\
(0.194)\end{array}$ \\
\hline Scale & $\begin{array}{c}2.332 * * * \\
(0.598)\end{array}$ & $\begin{array}{c}2.358^{* * * *} \\
(0.602)\end{array}$ & $\begin{array}{c}2.141 * * * \\
(0.629)\end{array}$ & $\begin{array}{c}2.108 * * * \\
(0.632)\end{array}$ & $\begin{array}{l}1.575^{\text {** }} \\
(0.669)\end{array}$ & $\begin{array}{l}1.580 * * \\
(0.647)\end{array}$ & $\begin{array}{c}2.128 * * * \\
(0.627)\end{array}$ & $\begin{array}{c}2.138 * * * \\
(0.724)\end{array}$ \\
\hline G_factor & $\begin{array}{c}-3.338 * * * \\
(0.753)\end{array}$ & $\begin{array}{c}-3.288^{* * *} \\
(0.754)\end{array}$ & $\begin{array}{c}-3.105 * * * \\
(0.795)\end{array}$ & $\begin{array}{c}-3.044 * * * \\
(0.796)\end{array}$ & $\begin{array}{c}-2.552 * * * \\
(0.809)\end{array}$ & $\begin{array}{c}-2.564 * * * \\
(0.788)\end{array}$ & $\begin{array}{c}-3.061 * * * \\
(0.791)\end{array}$ & $\begin{array}{c}-3.096^{* * * *} \\
(0.922)\end{array}$ \\
\hline T_fdi & $\begin{array}{c}-1.055^{* * * *} \\
(0.252)\end{array}$ & $\begin{array}{c}-1.075^{* * *} \\
(0.256)\end{array}$ & $\begin{array}{c}-0.888 * * * \\
(0.258)\end{array}$ & $\begin{array}{c}-0.844 * * * \\
(0.272)\end{array}$ & $\begin{array}{l}-0.578^{*} \\
(0.292)\end{array}$ & $\begin{array}{c}-0.621^{* *} \\
(0.271)\end{array}$ & $\begin{array}{c}-0.891 * * * \\
(0.257)\end{array}$ & $\begin{array}{c}-0.861^{* * *} \\
(0.311)\end{array}$ \\
\hline T_Rd & $1.386^{* * *}$ & $1.396^{* * *}$ & $1.451 * * *$ & $1.419 * * *$ & $1.347 * * *$ & $1.388^{* * *}$ & $1.444 * * *$ & $1.440 * * *$ \\
\hline
\end{tabular}




\begin{tabular}{ccccccccc} 
& $(0.242)$ & $(0.243)$ & $(0.259)$ & $(0.259)$ & $(0.251)$ & $(0.245)$ & $(0.255)$ & $(0.360)$ \\
_cons & 1.004 & 0.613 & -1.662 & -2.503 & -0.238 & 3.256 & -3.310 & $-4.111^{* *}$ \\
& $(1.803)$ & $(1.723)$ & $(2.081)$ & $(1.705)$ & $(3.617)$ & $(2.676)$ & $(2.484)$ & $(3.504)$ \\
Industry FE & YES & YES & YES & YES & YES & YES & YES & YES \\
Year FE & YES & YES & YES & YES & YES & YES & YES & YES \\
R-Squared & 75 & 75 & 75 & 75 & 75 & 75 & 75 & 75 \\
$\mathrm{~N}$ & 0.869 & 0.868 & 0.853 & 0.853 & 0.864 & 0.867 & 0.854 & 0.858 \\
\hline
\end{tabular}

472

\section{Industry Heterogeneity Analysis}

474 In order to investigate whether there is industry heterogeneity in the impact of participating in the $475 \mathrm{GVC}$ on $\mathrm{CO}_{2}$ emissions of China's equipment manufacturing industry, referring to the method of Peng and Kuang (2019), the equipment manufacturing industry is divided into high-tech and medium-tech equipment manufacturing industry. Empirical test of the impact of the forward and backward GVC production length on $\mathrm{CO}_{2}$ emissions has been conducted. High-tech industries include computer, electronic and optical product manufacturing, electrical equipment manufacturing industry and mechanical and equipment manufacturing industry, and medium-tech industries include fabricated metal products industry and transportation equipment manufacturing. The results are shown in Table 6 and Table 7.

Table 6 The impact of forward GVC production length on the $\mathrm{CO}_{2}$ emissions

\begin{tabular}{|c|c|c|c|c|c|c|c|c|}
\hline \multirow{2}{*}{ Variables } & \multicolumn{4}{|c|}{ High-tech equipment manufacturing industry } & \multicolumn{4}{|c|}{ Medium-technology equipment manufacturing industry } \\
\hline & (1) & (2) & (3) & (4) & (5) & (6) & (7) & $(8)$ \\
\hline \multirow{2}{*}{ PLv_GVC } & $-2.189 * *$ & & & & $1.153 * * *$ & & & \\
\hline & $(0.902)$ & & & & $(0.000132)$ & & & \\
\hline \multirow{2}{*}{ PLv_GVC_S } & & $-1.539 * *$ & & & & $1.183^{* * * *}$ & & \\
\hline & & $(0.749)$ & & & & $(0.000179)$ & & \\
\hline \multirow{2}{*}{ PLv_GVC_D } & & & $-1.725 * * *$ & & & & $0.612 * * *$ & \\
\hline & & & $(0.456)$ & & & & $(0.0000142)$ & \\
\hline \multirow{2}{*}{ PLv_GVC_F } & & & & $-1.858 * * *$ & & & & $0.520 * * *$ \\
\hline & & & & $(0.528)$ & & & & $(0.0000127)$ \\
\hline \multirow{2}{*}{ Policy } & $-0.636 * * *$ & $-0.700 * * *$ & $-0.730 * * *$ & $-0.821 * * *$ & $-0.182 * * *$ & $-0.169 * * *$ & $0.0685^{* * *}$ & $0.0382 * * *$ \\
\hline & $(0.191)$ & $(0.164)$ & $(0.125)$ & $(0.112)$ & $(0.00000637)$ & $(0.00000625)$ & $(0.00000330)$ & $(0.00000265)$ \\
\hline \multirow{2}{*}{ Scale } & $1.636^{*}$ & 1.412 & 1.414 & 1.505 & $0.222 * * *$ & $0.279 * * *$ & $0.168 * * *$ & $0.312 * * *$ \\
\hline & $(0.961)$ & (1.089) & (1.344) & (1.425) & $(0.00000780)$ & $(0.0000145)$ & $(0.0000371)$ & $(0.0000456)$ \\
\hline \multirow{2}{*}{ G_factor } & $-2.491 * * *$ & $-2.098^{* *}$ & -2.030 & -1.962 & $-1.175^{* * * *}$ & $-1.232 * * *$ & $-0.571 * * *$ & $-0.772 * * *$ \\
\hline & $(0.548)$ & $(0.836)$ & (1.271) & $(1.240)$ & $(0.0000220)$ & $(0.0000329)$ & $(0.0000287)$ & $(0.0000382)$ \\
\hline \multirow{2}{*}{ T_fdi } & 0.221 & 0.215 & 0.346 & 0.138 & $-0.0890 * * *$ & $-0.0804 * * *$ & $0.283 * * *$ & $0.251 * * *$ \\
\hline & $(0.277)$ & $(0.249)$ & $(0.281)$ & $(0.333)$ & $(0.00000681)$ & $(0.00000642)$ & $(0.00000299)$ & $(0.00000473)$ \\
\hline
\end{tabular}




\begin{tabular}{ccccccccc} 
T_Rd & $1.324 * * *$ & $1.400^{* * *}$ & $1.524 * * *$ & $1.557^{* * *}$ & $0.103^{* * *}$ & $0.112^{* * *}$ & $-0.0896^{* * *}$ & $-0.00307^{* * *}$ \\
& $(0.131)$ & $(0.166)$ & $(0.205)$ & $(0.202)$ & $(0.00000869)$ & $(0.0000112)$ & $(0.0000102)$ & $(0.0000138)$ \\
\multirow{2}{*}{ cons } & 3.250 & 2.635 & 4.072 & 3.603 & $2.205^{* * *}$ & $2.138^{* * *}$ & $2.735^{* * *}$ & $2.722^{* * *}$ \\
& $(3.644)$ & $(3.380)$ & $(3.238)$ & $(3.081)$ & $(0.0177)$ & $(0.0177)$ & $(0.0119)$ & $(0.0135)$ \\
Industry FE & YES & YES & YES & YES & YES & YES & YES & YES \\
Year FE & YES & YES & YES & YES & YES & YES & YES & YES \\
R-Squared & 0.907 & 0.906 & 0.913 & 0.912 & 0.997 & 0.997 & 0.998 & 0.998 \\
$\mathrm{~N}$ & 45 & 45 & 45 & 45 & 30 & 30 & 30 & 30 \\
\hline
\end{tabular}

485

Note: The robust standard errors in parentheses, ${ }^{* * *},{ }^{* *}$, and $*$ indicate significant at the level of $1 \%, 5 \%$, and $10 \%$ respectively.

Table 7 The impact of backward GVC production length on the $\mathrm{CO}_{2}$ emissions

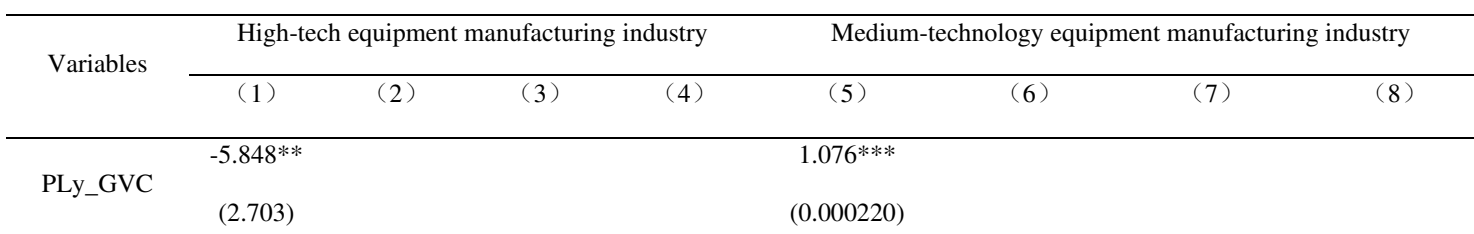

\begin{tabular}{lcc}
\hline PLy_GVC_S & $-3.995^{* *}$ & $1.235^{* * * *}$ \\
& $(2.037)$ & $(0.0000566)$ \\
\hline
\end{tabular}

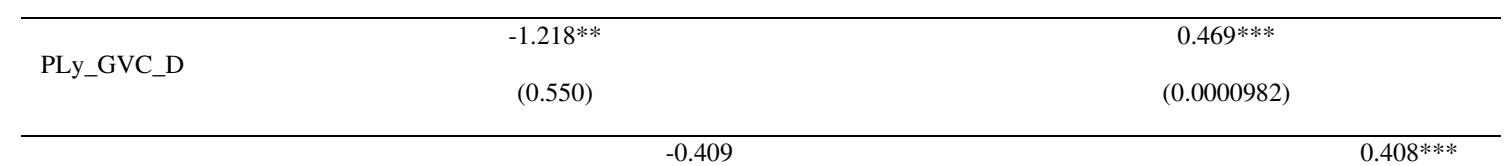

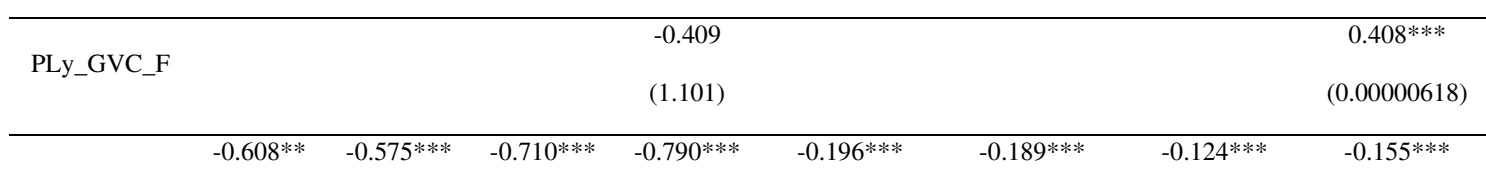

\begin{tabular}{|c|c|c|c|c|c|c|c|c|}
\hline \multirow{2}{*}{ Policy } & $-0.608 * *$ & $-0.575^{* * *}$ & $-0.710^{* * * *}$ & $-0.790 * * *$ & $-0.196^{* * *}$ & $-0.189^{* * * *}$ & $-0.124 * * *$ & $-0.155^{* * * *}$ \\
\hline & $(0.229)$ & $(0.0680)$ & $(0.150)$ & $(0.129)$ & $(0.0000137)$ & $(0.00000312)$ & $(0.00000112)$ & $(0.00000110)$ \\
\hline \multirow{2}{*}{ Scale } & 1.104 & 1.205 & 1.383 & 1.231 & $0.373 * * *$ & $0.283 * * *$ & $0.413 * * *$ & $0.265 * * *$ \\
\hline & (1.198) & $(0.772)$ & (1.173) & (1.544) & $(0.0000320)$ & $(0.0000138)$ & $(0.0000428)$ & $(0.0000222)$ \\
\hline \multirow{2}{*}{ G_factor } & -1.828 & $-2.027 * *$ & $-1.966 * *$ & -1.650 & -1.211 *** & $-1.089 * * *$ & $-1.176^{* * *}$ & $-1.124 * * *$ \\
\hline & (1.513) & $(0.857)$ & $(0.849)$ & (1.332) & $(0.0000333)$ & $(0.0000130)$ & $(0.0000283)$ & $(0.0000235)$ \\
\hline \multirow{2}{*}{ T_fdi } & -0.118 & -0.0149 & 0.392 & 0.266 & $-0.222^{*} * *$ & $-0.207 * * *$ & $-0.0861 * * *$ & $-0.0836^{* * *}$ \\
\hline & $(0.620)$ & $(0.295)$ & $(0.353)$ & $(0.434)$ & $(0.0000358)$ & $(0.0000103)$ & $(0.00000861)$ & $(0.00000653)$ \\
\hline \multirow{2}{*}{ T_Rd } & $1.437 * * *$ & $1.503 * * *$ & $1.420 * * *$ & $1.461 * * *$ & $0.0876^{* * * *}$ & $0.0712 * * *$ & $0.158 * * *$ & $0.134 * * *$ \\
\hline & $(0.310)$ & $(0.142)$ & $(0.163)$ & $(0.164)$ & $(0.00000777)$ & $(0.00000474)$ & $(0.0000232)$ & $(0.00000879)$ \\
\hline \multirow{2}{*}{ _cons } & 9.813* & $6.218 * * *$ & 3.193 & 1.698 & $1.675 * * *$ & $1.743 * * *$ & $2.321 * * *$ & $2.948 * * *$ \\
\hline & (5.166) & (1.069) & (3.891) & $(5.749)$ & $(0.00363)$ & $(0.00638)$ & $(0.00766)$ & $(0.00775)$ \\
\hline Industry FE & YES & YES & YES & YES & YES & YES & YES & YES \\
\hline Year FE & YES & YES & YES & YES & YES & YES & YES & YES \\
\hline R-Squared & 0.919 & 0.918 & 0.903 & 0.902 & 0.997 & 0.997 & 0.997 & 0.997 \\
\hline $\mathrm{N}$ & 45 & 45 & 45 & 45 & 30 & 30 & 30 & 30 \\
\hline
\end{tabular}

Note: The robust standard errors in parentheses, $* * *, * *$, and * indicate significant at the level of $1 \%, 5 \%$, and $10 \%$ respectively.

The first four columns of Table 6 show the estimated results of the impact of forward GVC production length of the high-tech industry on $\mathrm{CO}_{2}$ emissions, which is negative at a significant level of 
5\%; The first four columns in Table 7 indicate that the $\mathrm{CO}_{2}$ emissions of high-tech industries are subsequently reduced as the backward GVC production length is extended. The overall effect of the backward GVC production length on $\mathrm{CO}_{2}$ emissions is better than the forward GVC production length. The main reason for this phenomenon is that the production of high-tech industries in China's equipment manufacturing industry is mainly to provide high-level intermediate products to other countries. In this process, the level of production increases with deeply participating in GVC activities, and thus makes $\mathrm{CO}_{2}$ pollution in the production process continues to decrease. Due to the shortage of labor resources and the increase of basic production costs in China, the simple processing and production part of China's equipment manufacturing high-tech industry has begun to move to other developing countries, resulting in a stronger $\mathrm{CO}_{2}$ emission reduction effect caused by extending the length of backward GVC production length.

The last four columns in Table 6 show that the $\mathrm{CO}_{2}$ emissions of the medium-tech industry are affected by the extension of the forward GVC production length, that is, for every $1 \%$ increase of the forward GVC production length, $\mathrm{CO}_{2}$ emissions will increase by about $1 \%$. This is because the medium-technology industry in China's equipment manufacturing industry is still dominated by labor-intensive production. In the process of participating in the GVC, it has not completely separated from the low value-added and high $\mathrm{CO}_{2}$-emission production stage, and the $\mathrm{CO}_{2}$ emissions level of production is relatively high. The average growth rate of $\mathrm{CO}_{2}$ emissions from the transportation equipment manufacturing industry in 15 years was $8.36 \%$, the $\mathrm{CO}_{2}$ emissions increased by 458.57 million tons, and the average annual growth rate of the fabricated metal products industry reached $6.99 \%$. The coefficients of the backward GVC production length related indicators in the last four columns of Table 7 are all positive, indicating that the backward GVC production length also has a driving effect on the increase of $\mathrm{CO}_{2}$ emissions in the medium-tech industry. The reason is that the medium-tech industry in China's equipment manufacturing industry has a low position in the international division of production. This is because the fabricated metal product industry and transportation equipment manufacturing industry have higher requirements for precision parts, and the core technology manufacturing capabilities of China's equipment manufacturing industry are still weak, the change from basic core components, basic core technology and basic core materials to high-tech, high-end products and high-end components is very slow. China's medium-tech equipment manufacturing industry mainly provides final products to other countries in the form of OEM (Original Equipment Manufacturer). Therefore, the GVC participation mode based on backward linkage will generate more $\mathrm{CO}_{2}$.

It is worth noting that the regression results of the high-tech industries are in the same direction as the overall regression results, and will reduce $\mathrm{CO}_{2}$ emission, but the results of the medium-tech industries are opposite to the overall results. This may be because high-tech industries deepen the participation in the GVC by improving the level of research and development, while the increased participation in the GVC of the medium-tech manufacturing industry is at the expense of producing more resource-intensive products. China is committed to reducing resource-intensive production and encouraging high-tech $\mathrm{R} \& \mathrm{D}$ production activities, so that the $\mathrm{CO}_{2}$ emission reduction effect of high-tech industries is stronger than the $\mathrm{CO}_{2}$ promotion effect of medium-tech industries, which in the end will reduce the $\mathrm{CO}_{2}$ emissions of the whole equipment manufacturing industry. 


\section{Conclusions and policy implications}

534 Based on the decomposition framework of the GVC production length and the model of the impact of participating in $\mathrm{GVC}$ on $\mathrm{CO}_{2}$ emissions, this paper derives the core indicators and constructs a STIRPAT model of the impact of participating in $\mathrm{GVC}$ on $\mathrm{CO}_{2}$ emissions, clarifies the specific impact of different $\mathrm{GVC}$ participating modes on the $\mathrm{CO}_{2}$ emissions of the equipment manufacturing industry, and analyzes the industry heterogeneity of this impact. The main conclusions of the study are as follows:

First of all, the extension of the forward production length of the GVC can effectively reduce $\mathrm{CO}_{2}$ emissions. The extension of the forward simple GVC production length has the best effect, and the forward complex GVC production length has no effect on $\mathrm{CO}_{2}$ emissions; the $\mathrm{CO}_{2}$ emission reduction effect of the backward GVC production length and the backward simple GVC production length is significant, which is better than the result of the forward GVC production length, and the extension of the pure foreign $\mathrm{GVC}$ production length also has a slight $\mathrm{CO}_{2}$ emission reduction effect. It shows that the improvement of cleaner production technology in China's equipment manufacturing industry at the current stage mainly stays at the simple production stage of GVC, only reducing $\mathrm{CO}_{2}$ emissions in the processing and assembly links. Hence, the cleaner production technology of complex GVC production activities needs to be improved urgently.

Secondly, for high-tech industries, the extension of the forward and backward GVC production length will reduce $\mathrm{CO}_{2}$ emissions; while the extension of the forward and backward GVC production lengths of the medium-tech industry will increase $\mathrm{CO}_{2}$ emissions. This shows that the level of cleaner production in China's high-tech industries is increasing with the deepening of the participating degree of the GVC; however, the production of the medium-tech industries still relies on basic advantages such as abundant labor resources, and has been locked in the low-end link of the GVC. In addition, the high-tech industry has developed more vigorously, driving the overall $\mathrm{CO}_{2}$ emission reduction trend of the equipment manufacturing industry to improve. Therefore, China should implement an industry differentiation policy, improve the overall competitiveness of high-tech industries, and promote the realization of qualitative changes in low-tech industries (Chenyao et al. 2020).

Thirdly, policy regulations, factor structure and foreign investment can effectively reduce $\mathrm{CO}_{2}$ emissions, but the expansion of production scale and $\mathrm{R} \& \mathrm{D}$ investment will increase $\mathrm{CO}_{2}$ emissions. It shows that, in recent years, China's improvement in environmental regulations, the adjustment of factor structure and the introduction of foreign capital have brought positive $\mathrm{CO}_{2}$ emission reduction effects, but the problem of high- $\mathrm{CO}_{2}$ activities in the export intermediates production and inefficient use of $\mathrm{R} \& \mathrm{D}$ funds still exists.

Based on the above conclusions, the following policy implications are proposed:

Firstly, continue to deepen the degree of participating in GVC and move out of low-end production activities. In the context of participating in GVC, the extension of the GVC production length will bring great potential for $\mathrm{CO}_{2}$ reduction worldwide, especially in manufacturing sector (Rilong et al. 2020). It provides strong evidence for China's unswerving participation in the international division of labor and adherence to opening up. Therefore, China's equipment manufacturing industry should actively respond to the "Belt and Road" initiative, cooperate with countries along the "Belt and Road" in production activities, and undertake more high-value-added, low- $\mathrm{CO}_{2}$ production activities from 
developed countries. Transfer low-end production activities to other developing countries where resources and labor are cheaper. After that, China's equipment manufacturing industry can further extend the GVC production length, and be involved in the high-end production link of the GVC totally (Chenyao et al. 2020).

Secondly, maintain the advantages of intermediate production in simple GVC activities and improve the clean production technology level of complex GVC activities. At present, China's equipment manufacturing industry has made great progress in simple GVC production and has reached the requirements of cleaner production, but it still needs to improve the $\mathrm{CO}_{2}$ emission reduction effect of complex GVC activities. Accordingly, on the one hand, it is necessary to optimize the import quality of intermediate products through learning the manufacturing technology and processing technology of high-tech intermediate products, improve the level of intermediate products exported in complex GVC activities, and extend the GVC production length returning to the exporting countries. On the other hand, to encourage equipment manufacturing enterprises to "go global" means enterprises need to conduct in-depth cooperation with multinational companies in R\&D, design, brand building, etc., seek new path of participating in GVC with technological innovation to get out of the dilemma of "low-end lock-in" and extend the length of pure foreign GVC production length.

Thirdly, keep the clean production advantages of high-tech industries, accelerate the transformation and upgrading of medium-tech industries, and enable the equipment manufacturing industry to achieve the $\mathrm{CO}_{2}$ emissions reduction of the entire industry. For high-tech industries, while vigilant against the implementation of restrictions by countries with high-income, we should strive to achieve more advanced technological breakthroughs, seize the strategic position of high-end production links, and steadily move to the top of the GVC. The medium-tech industry needs to expand the production scale of high value-added intermediate products through the extensive introduction of advanced low- $\mathrm{CO}_{2}$ production technologies, reduce dependence on the export of pollution-intensive intermediate products, and gradually transform from the high- $\mathrm{CO}_{2} \mathrm{GVC}$ participation channels to high-tech channels. By this way, the entire equipment manufacturing industry will achieve $\mathrm{CO}_{2}$ emission reductions eventually.

Last but not least, continue to strengthen environmental control and foreign investment, improve the factor structure and R\&D expenditure utilization. (1) Environmental regulations have a guiding role in solving environmental problems. China should gradually raise the threshold of environmental control and improve the environmental pollution legal system. (2) The introduction of foreign capital has clearly helped the equipment manufacturing industry to upgrade clean technologies. China should continue to optimize its business environment and attract foreign investment in high-end technology industries. (3) The current factor structure of China's equipment manufacturing industry is still resource-oriented, and the $\mathrm{CO}_{2}$ emission increase effect of the expansion of production scale is obvious. The factor structure can be transformed to technology-intensive by increasing the skilled labor and R\&D personnel. (4) The government need to strictly supervise the destination of R\&D expenditures, allocate $R \& D$ expenditures reasonably, guide enterprises to use R\&D expenditures efficiently, and promote enterprises to increase investment in independent innovation, so that the enterprises of equipment manufacturing industry could climb to the higher level of participating in GVC through its own capabilities. 
Ethics approval and consent to participate Not applicable

Consent for publication Not applicable

Availability of data and materials The datasets used and analysed during the current study are available from the corresponding author on reasonable request.

Competing interests The authors declare no conflict of interest.

Funding information This research was funded by the National Social Science Fund of National

Office of Philosophy and Social Science of China (No. 17BJY071).

Author contributions Conceptualization: [Yan Li]; Methodology: [Yan Li]; Formal analysis and investigation: [Xinxin Xia]; Writing - original draft preparation: [Xinxin Xia]; Writing - review and editing: [Yan Li], [Qingbo Huang]; Funding acquisition: [Yan Li]; Resources: [Xinxin Xia];

\section{References}

Antweiler W, Copelan B. R, Taylor M. S (2001) Is free trade good for the environment. Am Econ Rev 4:877-908.

Cai L, Zhang Z, Zhu L (2020) Global Value Chain Embeddedness and Carbon Dioxide Emissions: An Empirical Study from China's Industrial Panel Data. J Int Trade 4:86-104.

Chang R, Yang L, Qian Z (2020) Does regional value chain participating help reduce the cost of value-added carbon emissions in my country? An empirical analysis based on manufacturing data. J Int Trade 5:117-131.

Chenyao Q, Jun S, Zhonghua C (2020) Can participating in global value chain drive green growth in China's manufacturing industry? J Clean Prod 268.

Chen C, Wang Y (2015) Vertical specialization and the upgrading dilemma of China's equipment manufacturing industry. Stud Sci Sci 33(8):1183-1192.

Chuanwang S, Zhi L, Tiemeng M, Runyong H (2019) Carbon efficiency and international specialization position: Evidence from global value chain position index of manufacture. Energy Policy 235-242.

Duan Y, Ji T, Yu T (2020) Reassessing pollution haven effect in global value chains. J Clean Prod $284: 124705$.

Edgar G. H (2020) Carbon fueling complex global value chains tripled in the period 1995-2012. Energy Econ 86.

Grossman G. M, Krueger A. B (1995) Economic growth and the environment. Q J Econ 2:353-377. 
Xu B, Yang L, Qian Z (2020) The impact of global value chain division of labor status on carbon emissions. Resour Sci 42(3):527-535.

Guy R A, Shaoyuan W, Yao L, Thierry B Y G, Diby F K, Akadje J E (2020) Dynamics between participation in global value chains and carbon dioxide emissions: empirical evidence for selected Asian countries. Environ Sci Pollut Res 27(9).

Hao X, Kejuan S, Xingwen T, Huimin B, Ming W (2020) Diversified carbon intensity under global value chains: A measurement and decomposition analysis. J Environ Manage 272.

Hofmann J, Guan D, Chalvatzis K, Huo H (2016) Assessment of electrical vehicles as a successful driver for reducing $\mathrm{CO}_{2}$ emissions in China. Appl Energy 184:995-1003.

Humphrey J, Schmitz H (2010) Governance in Global Value Chains. Ids Bulletin 3:19-29.

Huizheng L, Zhe Z, Kate H, Karolien D. B (2020) Can China reduce the carbon emissions of its manufacturing exports by moving up the global value chain? Res Int Bus Financ 51.

Kang S (2018) The status and influencing factors of China's manufacturing industry in the global value chain from the perspective of industry heterogeneity. Int Bus 4:74-85.

Lan T, Xia X (2020) Research on the embodied carbon of China-Europe manufacturing trade under the global value chain. J Cent South Univ (Soc Sci Ed) 26(4):111-123.

Lafang W, Youfu Y, Rui X, Shaojian W (2020) How global value chain participation affects China's energy intensity. J Environ Manage 260.

Li S (2019) Spatial econometric analysis of China's regional carbon emission intensity and its influencing factors. MA thesis, Beijing Jiaotong University Beijing.

Li Y, Yuan Y. The status of China's equipment manufacturing industry in the global value chain. J Int Econ Coop 4:10-13.

Liu H, Zhu G (2019) The impact of global value chain participating on the export technology complexity of China's equipment manufacturing industry: a study based on the heterogeneity of imported intermediate products. J Int Trade 8:80-94.

Lu Y, Chen S, Sheng B (2018) Will participating in the global value chain lead to the "low-end lock-in" made in China? Manag World 34(8):11-29.

Pan Q (2019) Research on the impact of global value chain participating on the transformation and upgrading of China's equipment manufacturing industry. World Econ Stud 9:78-96+135-136.

Peng X, Li B (2013) Research on the economic carbon emission effects of China's embedded manufacturing links from the perspective of global value chains. Financ. Trade Res 24(6):18-26.

Rilong F, An P, Xiaoli W, Qizhuo X (2020) How GVC division affects embodied carbon emissions in China's exports? Environ Sci Pollut Res. https://doi.org/10.1007/s11356-020-09298-8.

Stock T, Obenaus M, Kunz S, Kohl H (2018) Industry 4.0 as enabler for a sustainable development: A qualitative assessment of its ecological and social potential. Process Saf Environ Protect 118:254-267.

Sun H, Du X (2020) The impact of global value chain embeddedness and status on industrial carbon productivity. China Popul Res Environ 30(7):27-37.

Su B, Thomson E (2016) China's carbon emissions embodied in exports and their driving forces. Energy Econ 414-422.

Wang L (2014) The influence of integration into the global value chain on the international division of labor status of China's manufacturing industry. Stat Res 31(5):17-23.

Wang Y, Wang J, Yan J (2015) The dual effects of energy saving and emission reduction embedded in global value chains: an empirical study from China's industrial panel data. China Soft Sci $8: 148-162$. 
Peng H, Kuang X (2019) To what extent is China's manufacturing and producer services integrated-Based on the analysis and country comparison of the 2010-2014 international input-output table. J Int Trade 10:100-116.

Wei Q, Zhang C (2020) Research on China's manufacturing trade competitiveness from the perspective of $\mathrm{GVC}$ - Based on the comparison of total trade and value-added trade caliber. Price Mon $1: 55-63$.

Wu X, Pan A (2018) Does the technological effect reduce China's import and export embodied carbon emissions? Econ Surv 35(6):58-65.

Xie H (2018) Research on the Impact of Global Value Chain participating on China's Carbon Emissions and Productivity. PhD dissertation, Chongqing University Chongqing.

Xie H, Huang L, Liu D (2018) Does the global value chain participating increase the carbon productivity of China's manufacturing industry. J Int Trade 12:109-121.

Xu D, Yu F, Zhang M (2019) Can global value chain participating improve the low-carbon total factor productivity of Chinese industry? World Econ Stud 8:60-72+135.

Ying W, Zhaoxu W, Hashim Z (2021) Structural characteristics and evolution of the "international trade-carbon emissions" network in equipment manufacturing industry: international evidence in the perspective of global value chains. Environ Sci Pollut Res. https://doi.org/10.1007/S11356-021-12407-W.

Yu D, Tian S (2019) The influence mechanism of participating in global value chain on the transformation and upgrading of China's manufacturing industry. Reform 3:50-60.

Yuan Y, Qi J (2019) Research on the Impact of participating in Global Value Chains on Enterprise's Labor Income Share_-Based on the Measurement of the Length of Forward Production Chain. Ind Econ Res 5:1-12+38.

Yuan Y, Xi Q, Li G (2017) Research on the Mechanism and Effect of Industrial Correlation Level on the Evolution of Carbon Emissions__ An Empirical Analysis Based on Input-Output Data of 27 EU Countries. J Nat Resour32(5):841-853.

Zhang F, Gallagher KS (2016) Innovation and technology transfer through global value chains: evidence from China's PV industry. Energy Policy 94:191-203

Zhang R, Li A (2020) Measurement of the impact of integration into the global value chain on China's manufacturing productivity. Stat Decis 36(10):128-132.

Zhang H, Zhang Z, Sheng K (2018) The impact of global value chain division of labor status on China's manufacturing carbon emissions: an empirical study based on the STIRPAT model. Ecol Econ 34(4):25-29.

Zhang H, Wei X (2014) The green paradox may force emission reduction - the dual effect of environmental regulations on carbon emissions. China Popul Res Environ 24(9):21-29.

Zhao Y, Shi Q, Wu S (2020) The impact of participation in global value chains on the carbon intensity of China's export trade. J Beijing Inst Technol (Soc Sci Ed) 22(4):17-27.

Zhao L, Yang L (2020) The length of value chain production and the carbon emissions of China's manufacturing industry. J Technol Econ 39(5):156-162.

Zhi W, Shang-Jin W, Xinding Y, Kunfu Z (2017a) Characterizing Global Value Chains: Production Length and Upstreamness. NBER Working Paper 23261.

Zhi W, Shang-Jin W, Xinding Y, Kunfu Z (2017b) Measures of Participation in Global Value Chains and Global Business Cycles. NBER Working Paper 23222. 


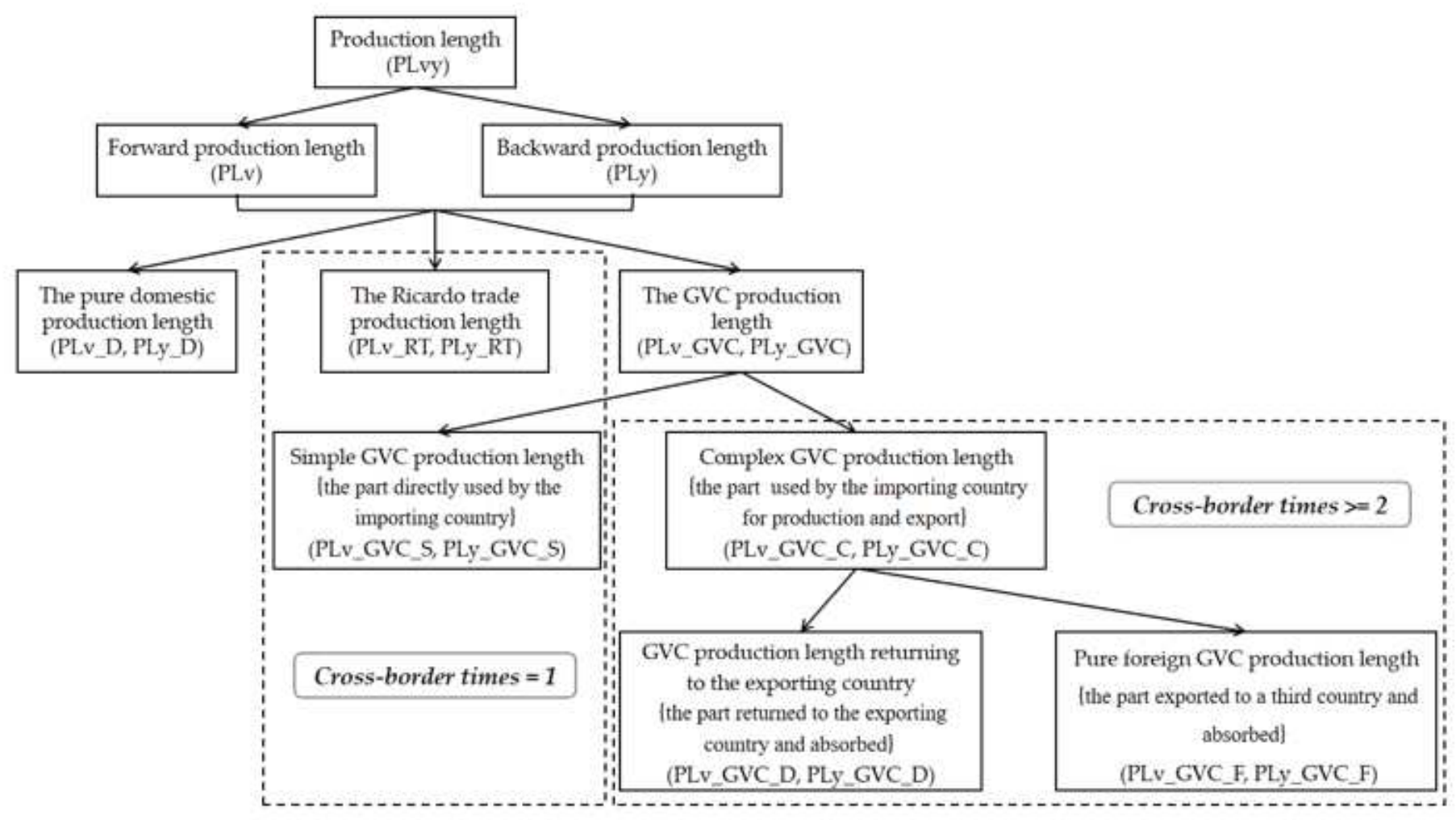

Figure 1

The decomposition model of production length

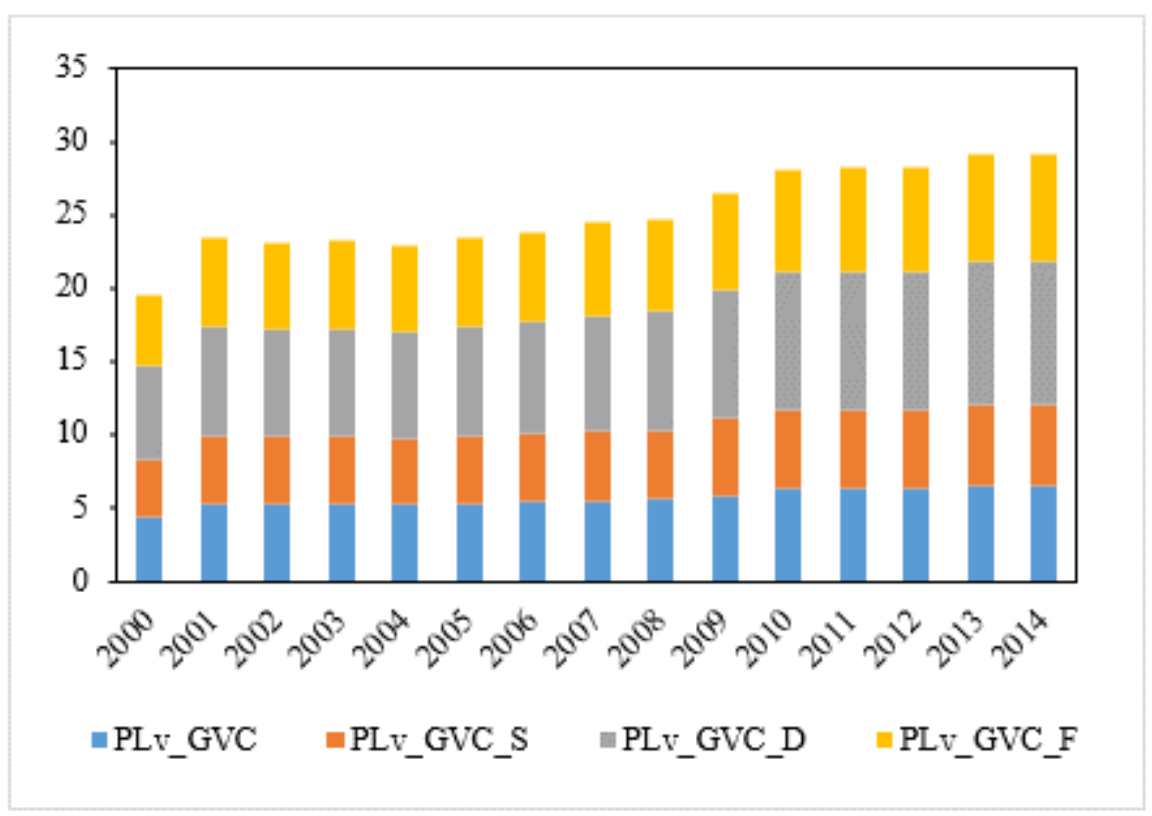

Figure 2 
The forward GVC production length of China's equipment manufacturing industry

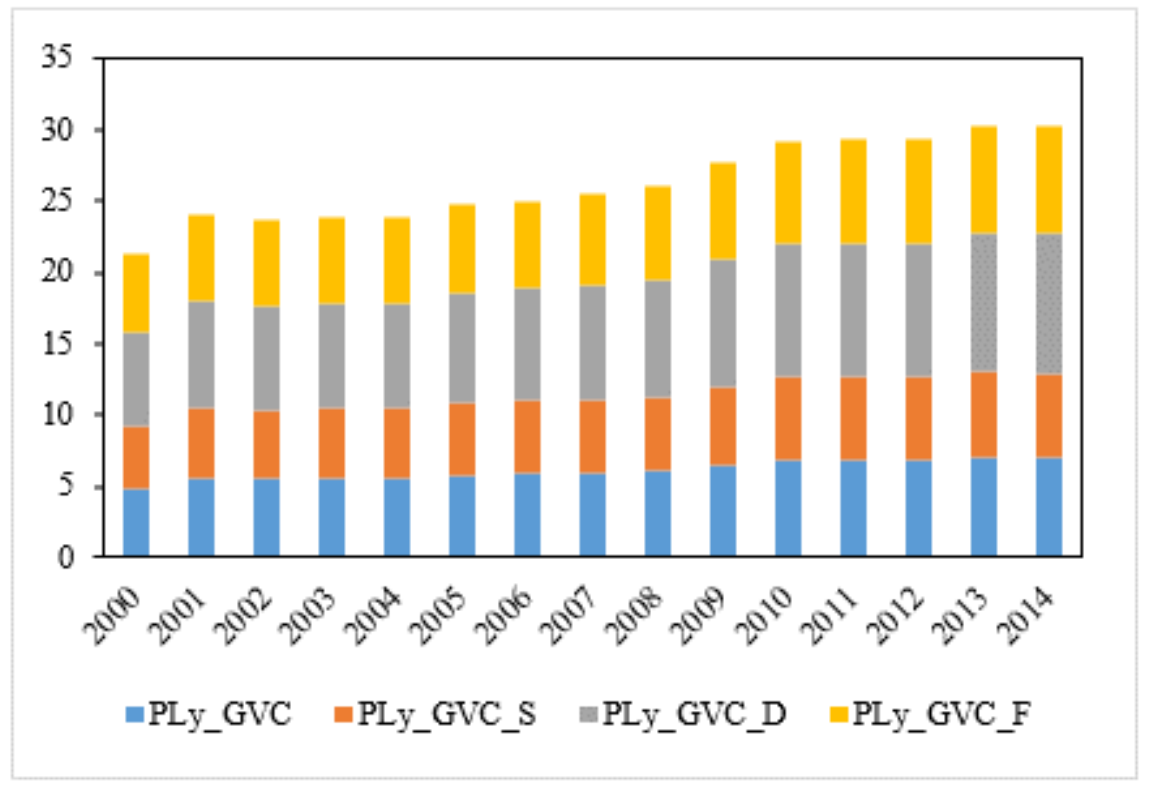

\section{Figure 3}

The backward GVC production length of China's equipment manufacturing industry

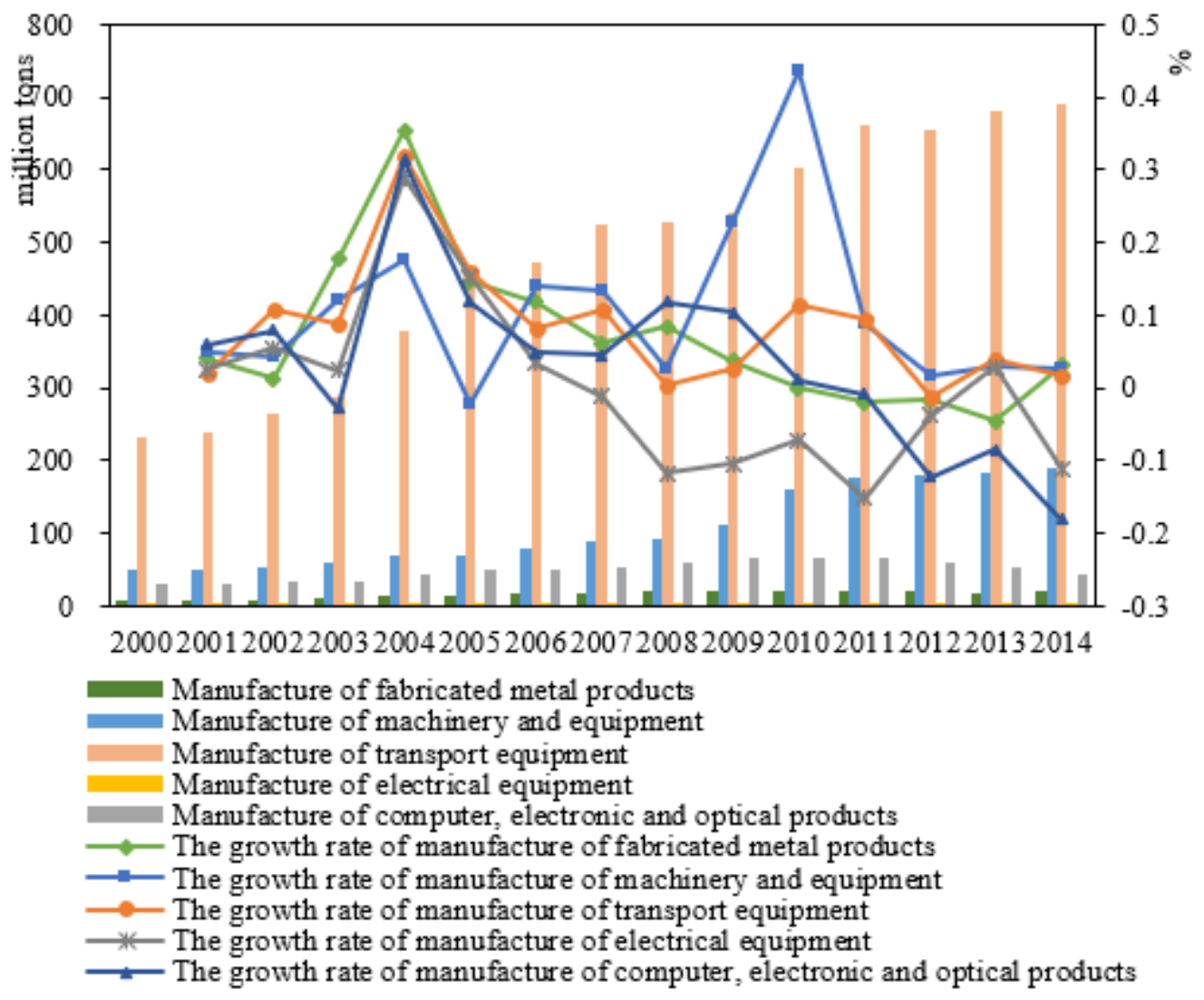

Figure 4 
The CO2 emission of China's equipment manufacturing industry from 2000 to 2014 